\title{
High-throughput screening discovers antifibrotic properties of haloperidol by hindering myofibroblast activation
}

Michael Rehman, ${ }^{1}$ Simone Vodret, ${ }^{1}$ Luca Braga, ${ }^{2}$ Corrado Guarnaccia, ${ }^{3}$ Fulvio Celsi, ${ }^{4}$ Giulia Rossetti, ${ }^{5}$ Valentina Martinelli, ${ }^{2}$ Tiziana Battini, ${ }^{1}$ Carlin Long, ${ }^{2}$ Kristina Vukusic, ${ }^{1}$ Tea Kocijan, ${ }^{1}$ Chiara Collesi, ${ }^{2,6}$ Nadja Ring, ${ }^{1}$ Natasa Skoko, ${ }^{3}$ Mauro Giacca, ${ }^{2,6}$ Giannino Del Sal, ${ }^{7,8}$ Marco Confalonieri, ${ }^{6}$ Marcello Raspa, ${ }^{9}$ Alessandro Marcello, ${ }^{10}$ Michael P. Myers, ${ }^{11}$ Sergio Crovella, ${ }^{3}$ Paolo Carloni, ${ }^{5}$ and Serena Zacchigna ${ }^{1,6}$

${ }^{1}$ Cardiovascular Biology, ${ }^{2}$ Molecular Medicine, and ${ }^{3}$ Biotechnology Development, International Centre for Genetic Engineering and Biotechnology (ICCEB), Padriciano, Trieste, Italy. ${ }^{4}$ Institute for Maternal and Child Health, IRCCS "Burlo Garofolo," Trieste, Italy. ${ }^{5}$ Computational Biomedicine Section, Institute of Advanced Simulation IAS-5 and Institute of Neuroscience and Medicine INM-9, Forschungszentrum Jülich GmbH, Jülich, Germany. ${ }^{6}$ Department of Medical, Surgical and Health Sciences, University of Trieste, Trieste, Italy. ${ }^{7}$ National Laboratory CIB, Area Science Park Padriciano, Trieste, Italy. ${ }^{8}$ Department of Life Sciences, University of Trieste, Trieste, Italy. ${ }^{9}$ National Research Council, CNRCampus International Development (EMMA-INFRAFRONTIER-IMPC), Monterotondo Scalo, Rome, Italy. ${ }^{10}$ Molecular Virology, and "Protein Networks Laboratories, International Centre for Genetic Engineering and Biotechnology (ICGEB), Padriciano, Trieste, Italy.

Fibrosis is a hallmark in the pathogenesis of various diseases, with very limited therapeutic solutions. A key event in the fibrotic process is the expression of contractile proteins, including $\alpha$-smooth muscle actin ( $\alpha$ SMA) by fibroblasts, which become myofibroblasts. Here, we report the results of a high-throughput screening of a library of approved drugs that led to the discovery of haloperidol, a common antipsychotic drug, as a potent inhibitor of myofibroblast activation. We show that haloperidol exerts its antifibrotic effect on primary murine and human fibroblasts by binding to sigma receptor 1 , independent from the canonical transforming growth factor- $\beta$ signaling pathway. Its mechanism of action involves the modulation of intracellular calcium, with moderate induction of endoplasmic reticulum stress response, which in turn abrogates Notch1 signaling and the consequent expression of its targets, including aSMA. Importantly, haloperidol also reduced the fibrotic burden in 3 different animal models of lung, cardiac, and tumor-associated fibrosis, thus supporting the repurposing of this drug for the treatment of fibrotic conditions.

Conflict of interest: The authors declare that no conflict of interest exists.

Copyright: (c) 2019 American Society for Clinical Investigation

Submitted: August 2, 2018 Accepted: March 14, 2019 Published: April 18, 2019

Reference information: /CI Insight. 2019;4(8):e123987. https://doi. org/10.1172/jici.nsight.123987.

\section{Introduction}

Fibrotic disorders, including cardiac fibrosis, idiopathic pulmonary fibrosis (IPF), chronic kidney fibrosis, liver cirrhosis, and systemic sclerosis, account for over $45 \%$ of deaths, with an increasing trend due to the overall aging of the human population worldwide (1). In addition, cancer-associated fibroblasts (CAFs) are emerging as key players in the growth and progression of a variety of tumors (2). Therefore, the development of safe and effective innovative therapies able to either prevent or reverse the progression of fibrosis is a relevant and unmet clinical need.

Even in organs that can undergo self-repair after injury, severe or chronic damage results in the formation of a fibrotic scar, which often occurs at the expense of regeneration. While the exact cellular and molecular mechanisms that distinguish physiological and self-limited wound healing from pathological nonresolving fibrosis are not clearly defined, a central role is certainly played by the differentiation of fibroblasts into myofibroblasts. The persistent activation of myofibroblasts stands as a key event shifting the reparative program towards fibrosis in several organs (3).

Progress has been made over the last years in the understanding of the signals and mechanisms responsible for myofibroblast activation. Matrix stiffness and mechanical stress in damaged tissues initiate a positive feedback loop between myofibroblasts and their surrounding environment that per- 
petuates the fibrotic response (4). Stiffness-activated fibroblasts start expressing $\alpha$-smooth muscle actin ( $\alpha \mathrm{SMA})$, which confers contractile activity to these cells, leading to extensive extracellular matrix (ECM) remodeling and further increases in matrix stiffness. Any tissue damage results in cell death that releases a variety of factors, which in turn induce the migration and activation of immune cells, including neutrophils, lymphocytes, and macrophages. All these cells express cytokines that exert strong profibrotic effects. In particular, transforming growth factor- $\beta$ (TGF- $\beta$ ) stimulates the migration of fibroblasts and their activation into myofibroblasts $(5,6)$.

Various molecules have so far been proposed and tested as antifibrotic agents. However, there is still no accepted effective treatment able to cure fibrotic diseases (7). Based on the assumption that fibrosis is the result of a chronic, unsolved inflammation, the most exploited therapy so far has been the use of corticosteroids (8-12). However, it is now clear that therapy with antiinflammatory drugs does not provide objective benefit (13-15). Moreover, these drugs are associated with a myriad of adverse effects that eventually worsen patients' quality of life.

Only recently, 2 drugs have been approved for the treatment of IPF, pirfenidone and nintedanib. Nintedanib was initially identified as a very potent antiangiogenic drug and later shown to inhibit multiple receptor tyrosine kinases (16). Pirfenidone was discovered in 1976 but did not receive approval until after 2011 by both the European Medicines Agency (EMA) and the Food and Drug Administration (FDA). Still, its exact mechanism of action remains unknown to date. Notably, the mechanism of action of both drugs probably entails major antiinflammatory properties $(17,18)$.

Very few approaches so far have aimed at interfering with myofibroblast activation to inhibit fibrosis. The observation that these cells rely on the production of antiapoptotic proteins, ensuring their survival, has paved the way to the development of small molecules, named Bcl-2 homology domain 3 (BH3) mimetics, targeting the antiapoptotic BCL-2 proteins. These drugs have been shown to induce myofibroblast apoptosis, thereby preventing and even reverting organ fibrosis in various experimental models (19-21). However, their translation to the clinical setting is far from becoming a reality, especially considering the pleiotropic activity of BCL-2 proteins in cell survival, with obvious side effects (3). Various other therapeutic strategies to inhibit myofibroblast function have been developed and tested in preclinical models, including kinase inhibitors (targeting RhoA kinase, extracellular signalregulated kinases [ERKs], c-Jun N-terminal kinases [JNKs], platelet-derived growth factor receptor $\beta$ [PDGFR $\beta]$, etc.), signaling pathway inhibitors (targeting TGF- $\beta$, Hedgehog, Notch, Wnt, endothelin-1, etc.), siRNAs, and microRNAs (22). Most of these proposed approaches have been developed based on in vitro experiments, while their therapeutic value in vivo and, more importantly, in patients, will require long preclinical and clinical experimentation.

The large gap between drug discovery and the effective implementation of new drug therapies in clinical settings warrants novel studies that take advantage of drugs that are already in development, on the market, or shelved because of lack of efficacy. Although drug repurposing typically occurs by accident, new technologies now enable the systematic evaluation of any drug on specific disease mechanisms.

Genetic screening has been used over the last several years to discover relevant pathways involved in myofibroblast activation (23-25). Here, we report the results of a high-content (HC), fluorescence-microscopy-based high-throughput screening (HTS), that led to the discovery of haloperidol, a common antipsychotic drug, as a potent inhibitor of myofibroblast activation. We showed that its mechanism of action involves binding to sigma receptor 1 (Sigmar1), modulation of intracellular calcium with moderate induction of endoplasmic reticulum (ER) stress response, eventually resulting in decreased expression of Notch1 and its targets, including aSMA. Importantly, the drug was also effective in 3 different mouse models of lung, cardiac, and tumor-associated fibrosis.

\section{Results}

Fibroblasts from different tissues are differentially prone to undergo myofibroblast differentiation. We compared the basal expression of $\alpha \mathrm{SMA}$, a typical marker for myofibroblast differentiation, in primary fibroblasts isolated from murine skin, lung, and heart. The purity of the fibroblast cultures is shown in Supplemental Figure 1; supplemental material available online with this article; https://doi.org/10.1172/jci.insight.123987DS1. The day after isolation and plating, $\alpha$ SMA mean cellular intensity was similar in all 3 cultures, while heart fibroblasts showed a lower number of $\alpha \mathrm{SMA}^{+}$cells than skin and lung fibroblasts (Supplemental Figure 2, A-C). We exposed cells to TGF- $\beta$ for 3 days and observed a marked differentiation to myofibroblasts, as 
shown by the $80 \%$ increase in the number of $\alpha \mathrm{SMA}^{+}$cells in all conditions (Supplemental Figure 2, D and E). Mean $\alpha$ SMA intensity was also significantly increased in all cell types, with cardiac fibroblasts showing the best response, resulting in a 2-fold increase in aSMA expression (Supplemental Figure 2, D and F).

Besides responding to TGF- $\beta$, primary fibroblasts differentiate to myofibroblasts spontaneously in culture $(26,27)$, although at a slower rate. We took advantage of $\alpha$ SMA-RFP/COLL-EGFP transgenic mice, which simultaneously express red fluorescent protein (RFP) under the control of the $\alpha$ SMA promoter and enhanced green fluorescent protein (EGFP) under the control of the collagen $\alpha 1$ (I) promoter (28). Fibroblasts from skin, lung, and heart of $\alpha$ SMA-RFP/COLL-EGFP mice were kept in culture for 9 days and both green and red fluorescence were monitored as surrogate markers for myofibroblast differentiation. Skin fibroblasts exhibited the highest response, showing a 6-fold increase in the aSMA expression already evident on day 5 and reaching a plateau over the following days, while lung fibroblasts underwent a more modest differentiation, reaching a 3-fold increase in aSMA expression at day 9 (Supplemental Figure 3, A-D). Cardiac fibroblasts exhibited an intermediate response, showing a progressive increase in $\alpha \mathrm{SMA}$ expression, which peaked at day 9 and reached an almost 5-fold increase (Supplemental Figure 3, E and F).

Collectively, these results indicate that fibroblasts from various tissues are differentially prone to differentiate into myofibroblasts, both spontaneously and upon TGF- $\beta$ stimulation. Because of their intermediate phenotype, we chose cardiac fibroblasts for our further HC-HTS studies aimed at identifying both positive and negative modulators of $\alpha \mathrm{SMA}$ expression.

Modulators of aSMA expression in myofibroblasts identified by HC-HTS. We performed HC-HTS in cardiac fibroblasts, using a library of 640 drugs approved by the FDA, categorized into 5 groups: cardiovascular agents, neuropsychiatric agents, antibiotics, antiinflammatory agents, and others (Figure 1A). Fibroblasts from adult $\alpha$ SMA-RFP/COLL-EGFP mice were seeded into 384-well plates (1200 cells/well), and 24 hours later each compound was spotted on top of the cells at the final concentration of $10 \mu \mathrm{M}$. After an additional 48 hours, the cells were fixed and nuclei stained with Hoechst 33342 (on average 375 cells were analyzed per experimental condition). Results of the screening for the 598 chemical compounds that did not decrease cell viability are reported in Figure 1B and Supplemental Table 1. The levels of $\alpha$ SMA expression were assessed by automated measurement of mean cell intensity in the red channel. Two independent replicates of the screening were performed (Pearson's $r=0.474$ ) and the $Z$ score was calculated from the average of both replicates (Figure 1B). This screening identified 5 compounds that significantly decreased $(Z$ score $\leq-1.96 ; P$ $\leq 0.05$ ) and 12 compounds that significantly increased $(Z$ score $\geq 1.96 ; P \leq 0.05) \alpha S M A$ expression. Representative images of the most effective compounds modulating $\alpha$ SMA expression are reported in Figure $1 C$.

Next, we individually validated the 5 hits that inhibited aSMA expression (dexamethasone, haloperidol, apomorphine, retinoic acid, and clodronate disodium) on cardiac fibroblasts from CD1 mice by staining for $\alpha$ SMA with specific antibodies (Figure 1D). As expected, TGF- $\beta$ upregulated $\alpha$ SMA expression ( $>2$-fold increase), whereas all the tested drugs reduced TGF- $\beta$-induced $\alpha$ SMA levels, with the most potent effect exerted by dexamethasone and haloperidol (Figure 1, D and E). Dexamethasone is one of the most common corticosteroid drugs, which are currently being considered to reduce the inflammatory burden in various fibrotic diseases (29-31), thereby confirming the successful setup of the screen. However, as mentioned above, the clinical use of these drugs is fraught with major side effects, including osteoporosis, hypertension, diabetes, and weight gain. Therefore, we focused our interest on the second-most-potent drug, haloperidol (4-[4-(4-chlorophenyl)-4-hydroxy-1-piperidyl]-1-[4-fluorophenyl]-butan-1-on).

Fibroblasts from different tissues respond to haloperidol in a similar manner. To determine the lowest effective concentration of haloperidol able to downregulate $\alpha$ SMA levels, we measured cell viability in a dose-dependency test on cardiac fibroblasts. Supplemental Figure 4A shows the dose-response curve for viability, with a half maximal inhibitory concentration $\left(\mathrm{IC}_{50}\right.$ ) value of $2.93 \mu \mathrm{g} / \mathrm{ml}$, corresponding to a $7.8 \mu \mathrm{M}$ concentration. Next, we tested 4 different haloperidol doses, selected in the nontoxic range $(<7.8 \mu \mathrm{M})$, for their ability to reduce $\alpha$ SMA expression upon TGF- $\beta$ treatment. As shown in Supplemental Figure 4, B and C, there was an inverse relationship between the dose of haloperidol and the intensity of $\alpha \mathrm{SMA}$ expression, which reached its minimum level at $3 \mu \mathrm{M}$. No further decrease in $\alpha \mathrm{SMA}$ expression was seen at $6 \mu \mathrm{M}$. Therefore, we selected the $3-\mu \mathrm{M}$ dose for the subsequent experiments, in line with previous studies showing an effect of haloperidol on fibroblast proliferation (32). This dose resulted in a marked inhibition of TGF- $\beta$-induced overexpression of aSMA, as detected by Western blotting (Figure 1, F and G) and quantitative PCR (Acta2), as well as of other markers associated with myofibroblast activation, including collagen 1A1 (Colla1), fibronectin 1 (Fn1), and periostin (Postn) (Figure 1H). We then checked whether haloperidol exerted a similar activity in fibro- 
A
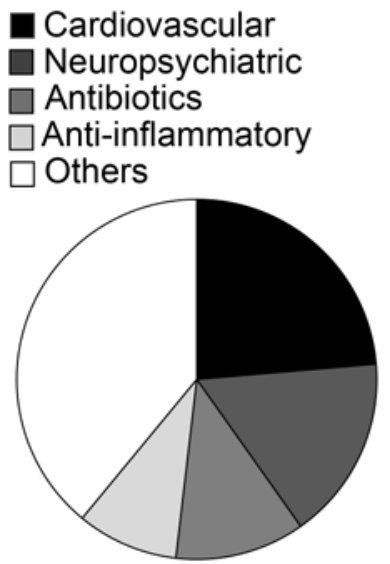

D

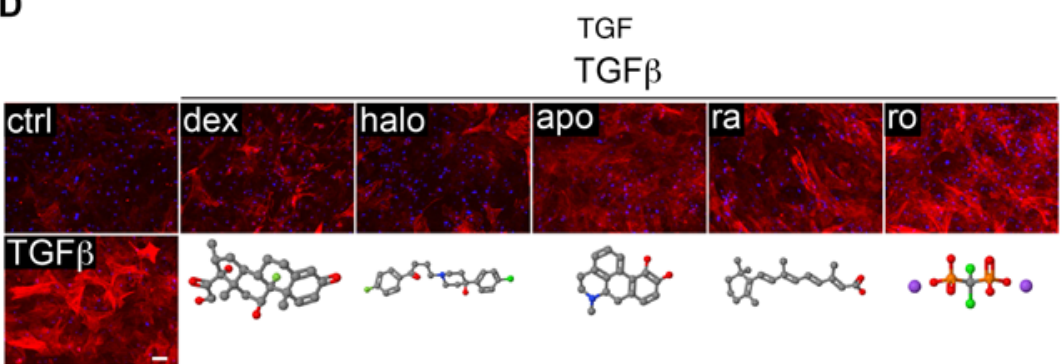

aSMAHoechst

$\mathbf{F}$

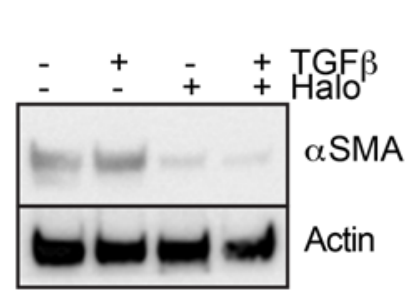

B

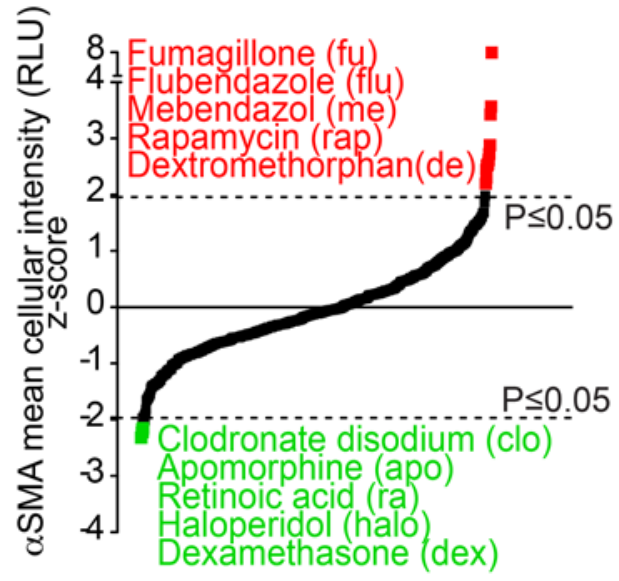

G

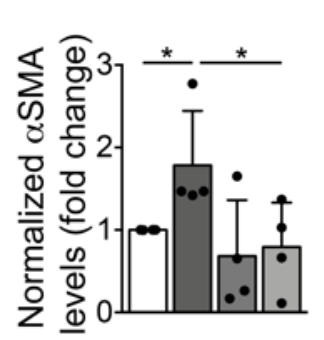

H
C
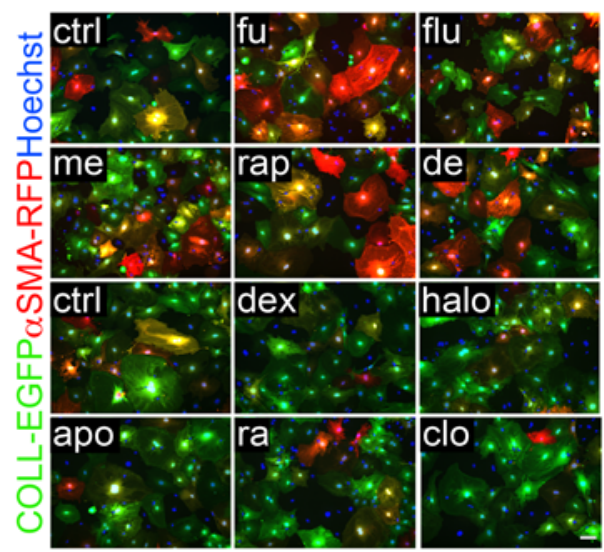

E
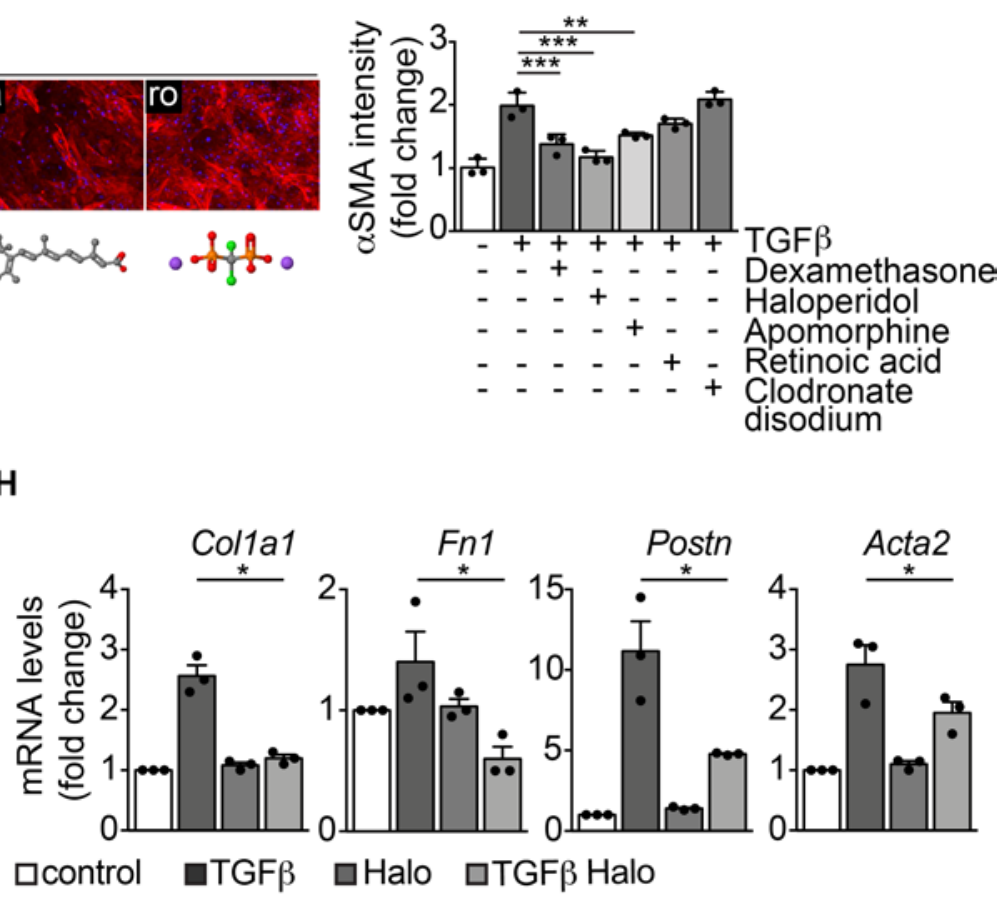

Figure 1. High-throughput screening identifies several modulators of $\alpha$ SMA expression in myofibroblasts. (A) Pie chart showing the main categories of the 640 FDA-approved drugs included in the library. (B) Results of the high-throughput screening shown as the $Z$ score of the $\alpha$ SMA mean cellular intensity in $\alpha S M A-R F P / C o L L-E G F P$ fibroblasts, treated with each drug at 48 hours of culture. Top hits upregulating $\alpha$ SMA expression are indicated in red, whereas those downregulating $\alpha$ SMA expression are indicated in green. (C) Representative images of $\alpha$ SMA-RFP/COLL-EGFP fibroblasts exposed to the indicated drugs (abbreviations are explained in panel B). Green fluorescence indicates collagen expression (COLL-EGFP), whereas red fluorescence indicates $\alpha$ SMA expression ( $\alpha$ SMARFP). Nuclei are stained blue with Hoechst. (D) Cardiac fibroblasts treated with TCF- $\beta$ alone and in combination with various drugs, stained red with anti- $\alpha$ SMA antibodies. Nuclei are stained blue with Hoechst. The chemical structure of each drug is shown under the corresponding cell picture. Atom color assignment: gray, carbon; red, oxygen; green, halogen; blue, nitrogen; orange, phosphorus; purple, sodium. (E) Quantification of the $\alpha$ SMA mean fluorescence intensity upon treatment with TCF- $\beta$ alone and in combination with the indicated drugs ( $n=3 / \mathrm{gp})$. (F) Western blot showing the expression of $\alpha$ SMA in primary cardiac fibroblasts treated with TGF- $\beta$, haloperidol (3 $\mu \mathrm{M})$, or their combination. Actin is shown as loading control. (G) Quantification of $\alpha$ SMA levels in primary cardiac fibroblasts treated with TGF- $\beta$, haloperidol $(3 \mu \mathrm{M})$, and their combination ( $n=4 / \mathrm{gp})$. (H) Expression levels of Col1a1, Fn1, Postn, and Acta2 upon treatment with TGF- $\beta$, haloperidol, and their combination ( $n=3 /$ gp). Values in $\mathbf{E}, \mathbf{G}$, and $\mathbf{H}$ are mean \pm SEM. ${ }^{*} P<0.05,{ }^{* *} P<0.01,{ }^{* * *} P<0.001$ by unpaired $t$ test with Welch's correction. Scale bars in C and D: $50 \mu \mathrm{m}$.

blasts derived from skin and lung and found that in both cases TGF- $\beta$ treatment resulted in the upregulation of $\alpha \mathrm{SMA}$, which was significantly reduced by treatment with haloperidol (Supplemental Figure 4, D-G).

Consistent with previous studies (33), we observed that TGF- $\beta$ exerted a mitogenic activity on primary cardiac fibroblasts, as evidenced by their increased cell number and percentage of $\mathrm{Ki}-67^{+}$cells (Figure 2, A-C). Haloperidol effectively inhibited these effects, reverting both parameters back to basal levels (Figure 
2, A-C). Next, we investigated the effect of haloperidol on fibroblast migratory ability using a scratchwound assay. We found that TGF- $\beta$ accelerated, whereas haloperidol impaired, wound closure (Figure 2, D and E). Finally, we performed a collagen gel contraction assay to measure the force generated by myofibroblasts, resulting in the contraction of the collagen matrix. As expected, TGF- $\beta$ clearly promoted gel contraction, while the cotreatment with haloperidol markedly inhibited this effect (Figure 2, F and G). Collectively, these data indicate that haloperidol inhibits multiple TGF- $\beta$-induced features typical of activated myofibroblasts, reverting them back to a quiescent phenotype.

Sigmar1 is the mediator of haloperidol activity in fibroblasts. Haloperidol is effectively used in clinics as a potent antipsychotic drug, blocking the dopamine D2 receptor (DRD2) in the central nervous system (34, 35). Therefore, we checked the expression of DRD2 in primary fibroblasts from murine skin, lung, and heart and compared it to that in the SH-SY5Y neuronal cell line. None of these fibroblast types expressed the receptor, whereas it was abundantly expressed by the neuronal cells, as expected (Figure 3A). Interestingly, haloperidol also binds and antagonizes the activity of Sigmar1, which is reported to be expressed by a variety of cell types (36). Consistently, we found it abundantly expressed by skin, lung, and heart fibroblasts at both mRNA and protein levels (Figure 3, A and B). Interestingly, haloperidol was able to upregulate its own receptor, Sigmar1, in cardiac, skin, kidney, and lung fibroblasts (Figure 3C and Supplemental Figure 5).

The crystal structure of Sigmar1 was recently reported in combination with its ligands, $N$-(1-benzylpiperidin-4-yl)-4-iodobenzamide (PD 144418), 3-(4-methylphenyl)-5-(1-propyl-3,6-dihydro-2H-pyridin-5-yl)-1,2oxazole, and $\mathrm{N}$-(1-benzylpiperidin-4-yl)-4-iodobenzamide (4-IBP) (37). Both structures (5HK1 and 5HK2) revealed a trimeric architecture for Sigmar1, in which ligands bound to the same hydrophobic cavity. Therefore, we hypothesized that haloperidol may also bind to this region. To test this hypothesis, we performed a screening of the receptor surface to identify druggable binding sites in the deposited structures. In both cases, the first 3 high-ranked binding sites corresponded to the 3 hydrophobic cavities in each monomer, confirming our hypothesis (see Supplemental Table 2). Despite the similar binding mode of the ligands (Supplemental Figure 6A) and the equivalent geometry of the binding sites in each monomer, we could identify significant differences in binding residues, size, and druggability of each site (Supplemental Figure 6B). Three different configurations of haloperidol were considered at $\mathrm{pH} 7.0 \pm 2$, labeled as $\mathrm{A}, \mathrm{B}$, and $\mathrm{C}$ (Supplemental Figure 6C). These were docked on each of the 6 identified binding cavities (Supplemental Figure 6D). In each binding site the highest- and the lowest-ranked haloperidol conformations, according to both Glide Score and Emodel score $(38,39)$, are A and C, respectively (Supplemental Table 3). In its highest-ranked binding pose, haloperidol forms interactions with almost all the residues of the binding cavity (Supplemental Figure $6 \mathrm{E}$ and Supplemental Table 4), in agreement with those reported by Yano et al. (40).

To demonstrate that Sigmar1 is the receptor mediating the inhibitory effect of haloperidol on $\alpha \mathrm{SMA}$ expression, we silenced its expression using 4 different short hairpin RNAs (shRNAs), using a scrambled sequence of the first shSigmar1 as a control. All shRNAs effectively reduced the levels of Sigmar1 expression in cardiac fibroblasts at both the mRNA and protein level (Figure 3, D and E) as well as in the NIH3T3 fibroblast cell line (Supplemental Figure 7A). As expected, the control scramble shRNA did not affect the activity of haloperidol. In contrast, silencing of Sigmar1 potently inhibited the ability of haloperidol to reduce the expression of $\alpha$ SMA upon treatment with TGF- $\beta$, indicating that Sigmar1 is required by haloperidol to inhibit myofibroblast activation (Figure 3, F and G, and Supplemental Figure 7, B and C).

Modulation of $\alpha S M A$ expression by haloperidol involves an increase in intracellular calcium, mild ER stress, and eventual downregulation of the Notch pathway. TGF- $\beta$ signaling is the main driver of fibrosis in most, if not all, organs, via activation of both canonical (Smad-dependent) and noncanonical pathways. To determine whether the effect of haloperidol impinges on either of these pathways, we first performed a CAGA-Luc reporter assay, in which luciferase expression is induced upon binding of Smad2/3 to the CAGA box. As shown in Figure 4A, TGF- $\beta$ resulted in an almost 30 -fold increase in reporter activity, which was not reduced by haloperidol. This was a first indication that haloperidol acts independently of the TGF- $\beta$ canonical pathway. In addition, we tested the subcellular localization of SMAD2/3, which translocates to the nucleus upon TGF- $\beta$ stimulation. As shown in Figure $4, \mathrm{~B}$ and $\mathrm{C}$, haloperidol did not affect this translocation, again ruling out an effect of this drug on the TGF- $\beta$ canonical pathway. Finally, we tested the phosphorylation of SMAD2 upon haloperidol treatment and again found that haloperidol did not significantly change the levels of TGF- $\beta$-induced p-SMAD2 in either cardiac fibroblasts or NIH3T3 cells (Figure 4D). Collectively, these data imply that haloperidol does not act through the canonical TGF- $\beta$ pathway. 
A

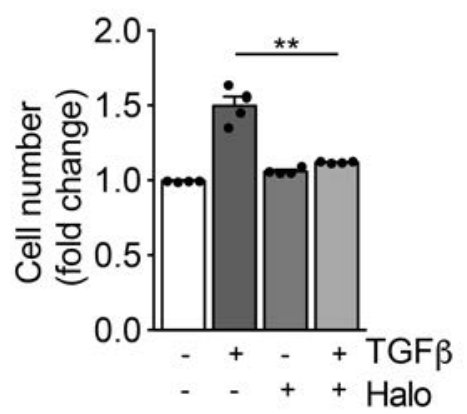

D

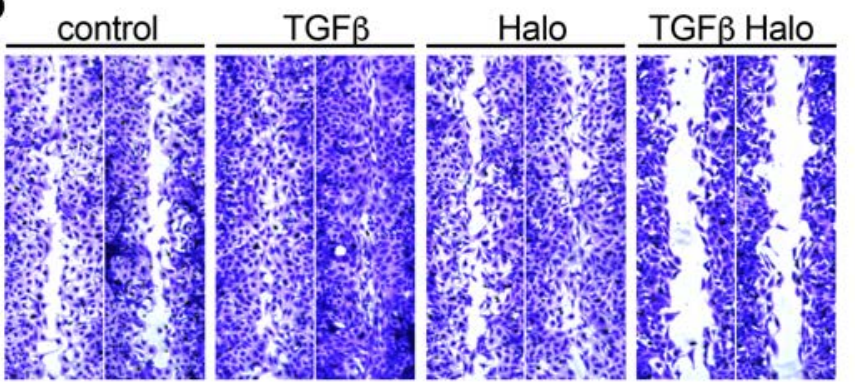

$\mathbf{F}$

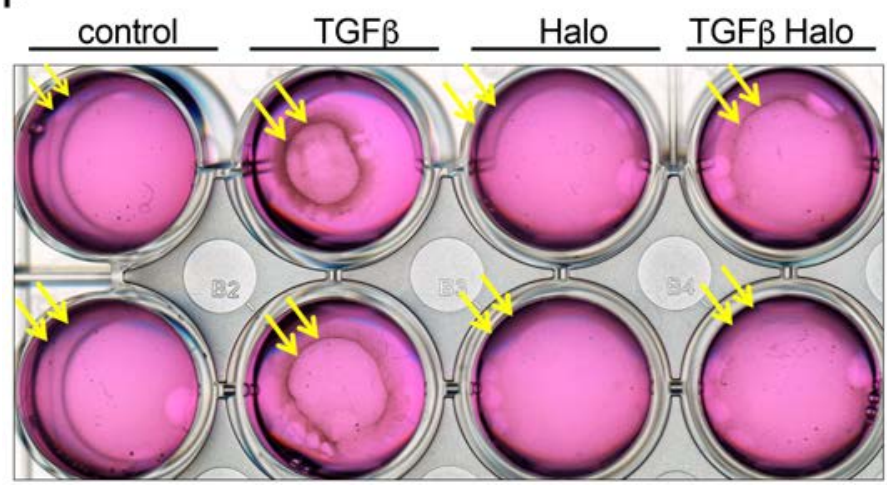

B

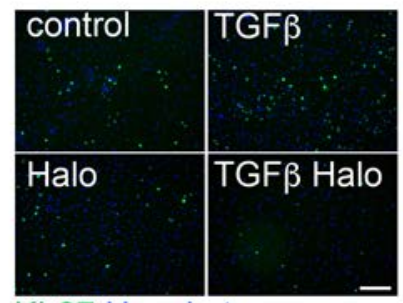

Ki-67 Hoechst
C

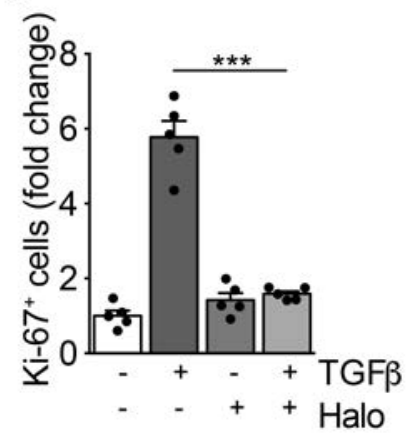

E

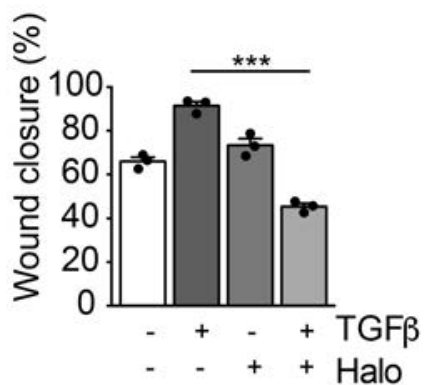

G

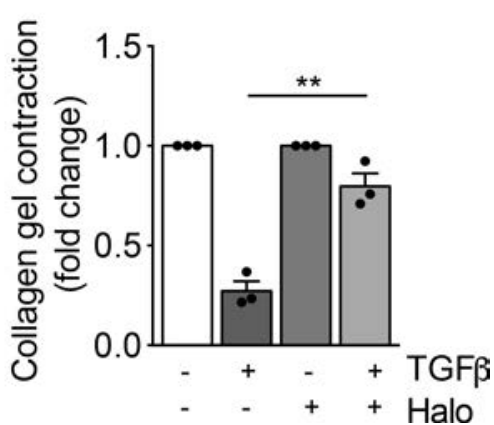

Figure 2. Haloperidol inhibits myofibroblast proliferation, migration, and contraction. (A) Quantification of cardiac fibroblast growth upon treatment with TCF- $\beta$, haloperidol, and their combination for 72 hours $(n=4 / \mathrm{gp})$. (B) Representative images of Ki-67 immunofluorescence in primary cardiac fibroblasts treated with TGF- $\beta$, haloperidol, and their combination for 72 hours. Nuclei are stained blue with Hoechst. Scale bar: $50 \mu \mathrm{m}$. (C) Quantification of $\mathrm{Ki}-67^{+}$cells in cardiac fibroblasts treated with TCF- $\beta$, haloperidol, or their combination for 72 hours ( $\left.n=5 / g p\right)$. (D) Representative images of scratch-wound assay using primary cardiac fibroblasts treated with TCF- $\beta$, haloperidol, and their combination and stained with crystal violet. (E) Quantification of wound closure by primary cardiac fibroblasts treated with TCF- $\beta$, haloperidol, or their combination at 24 hours ( $n=3 / g p$ ). (F) Representative images of collagen gel contraction by embedded cardiac fibroblasts treated with TCF- $\beta$, haloperidol, and their combination. (G) Quantification of gel contraction by primary cardiac fibroblasts treated with TCF- $\beta$, haloperidol, or their combination at 24 hours $(n=3 / \mathrm{gp})$. Values in $\mathbf{A}, \mathbf{C}, \mathbf{E}$, and $\mathbf{G}$ are mean \pm SEM. ${ }^{* *} P<0.01,{ }^{* * *} P<$ 0.001 by unpaired $t$ test with Welch's correction.

Because previous reports showed that haloperidol regulates intracellular calcium levels via Sigmar1 in neuronal and epithelial cells $(41,42)$, we assessed whether haloperidol also induced any variation in cytosolic calcium in fibroblasts. Live imaging of Fluo4-labeled single cells indicated that haloperidol rapidly increased the intracellular levels of calcium (Figure 4, E and F, and Supplemental Video 1). Similar results were obtained when Fluo4 fluorescence was measured in a plate-based assay both in cardiac fibroblasts and NIH3T3 cells (Figure 4G). The role of Sigmar1 in mediating this change in intracellular calcium levels was confirmed by performing the Fluo4 fluorescence assay in cardiac fibroblasts stably expressing the 4 shRNAs targeting Sigmar1. As shown in Supplemental Figure 8, Sigmar1 silencing reduced the increase in intracellular calcium observed upon haloperidol treatment. The curve showing intracellular calcium levels upon haloperidol administration imitated the one obtained by the treatment of the same cells with thapsigargin, an inhibitor of the calcium pump SERCA and inducer of ER stress $(43,44)$, as shown in Figure $4 \mathrm{H}$. 
A

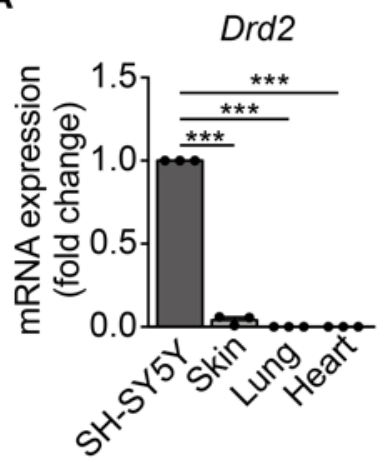

D

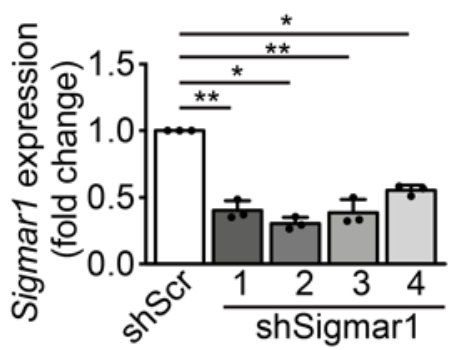

E

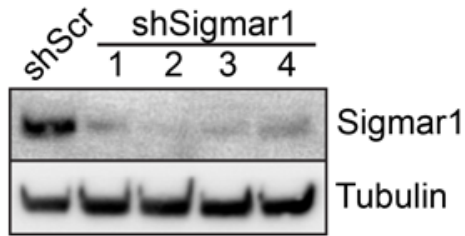

B

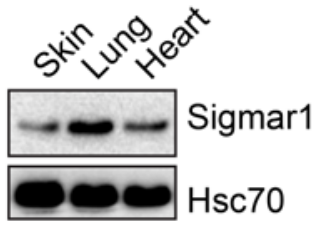

C

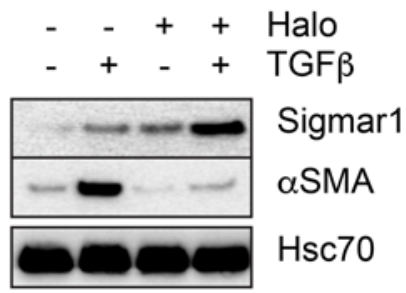

$\mathbf{F}$

TGF $\beta$

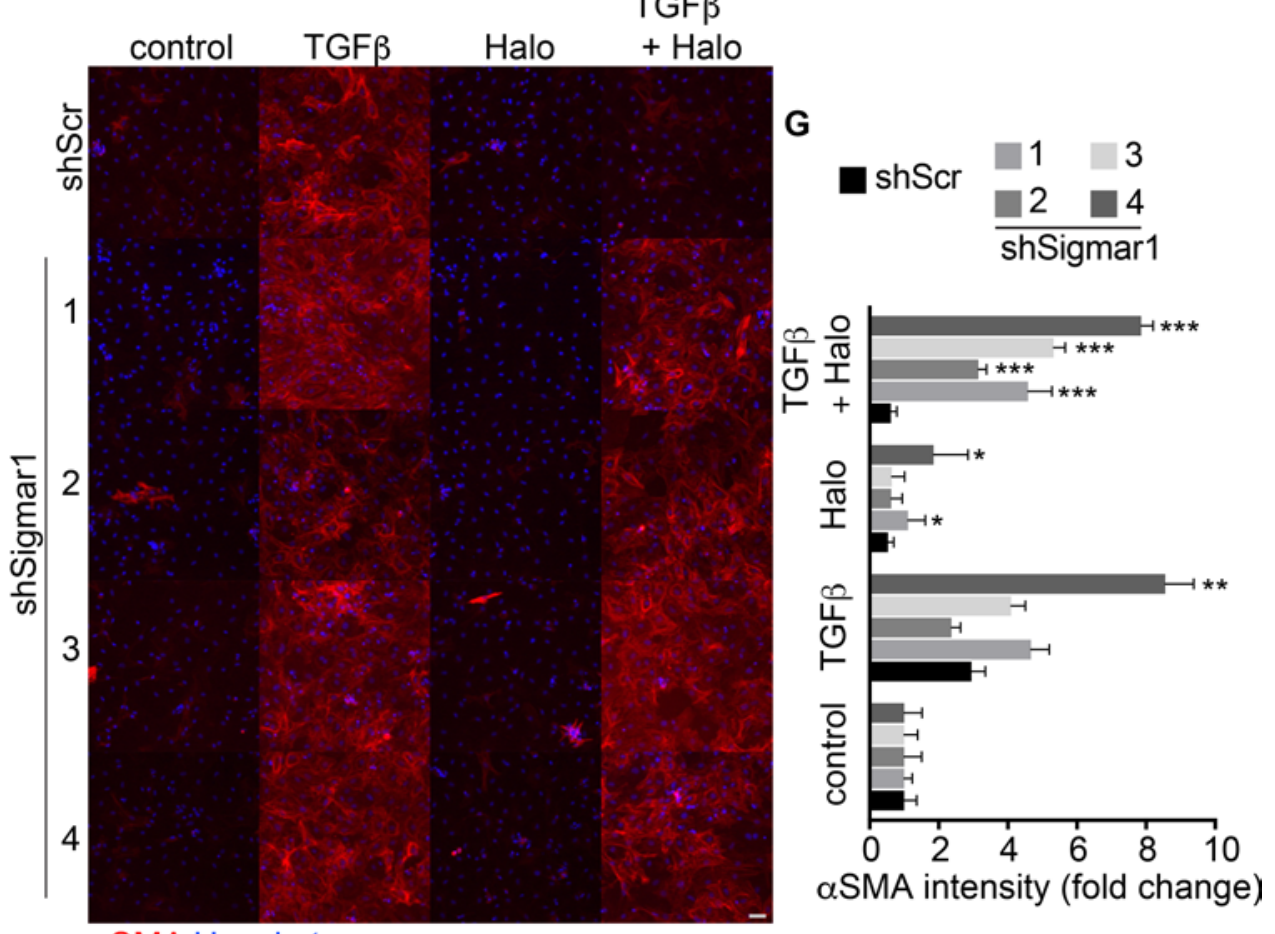

aSMA Hoechst

Figure 3. Sigmar1 mediates haloperidol activity in fibroblasts. (A) Relative mRNA expression of dopamine receptor D2 (Drd2) and sigma receptor 1 (Sigmar1) in SH-SY5Y neuronal cells, skin, lung, and heart fibroblasts $(n=3 / \mathrm{gp})$. (B) Western blot for Sigmar1 in skin, lung and cardiac fibroblasts. Hsc70 is shown as loading control run in parallel with Sigmar1. (C) Western blot showing the expression of $\alpha$ SMA and Sigmar1 in cardiac fibroblasts upon treatment with TCF- $\beta$, haloperidol, or their combination. Hsc70 is shown as loading control run in parallel. (D) Quantitative real-time PCR showing the level of Sigmar1 expression upon delivery of 4 specific shRNAs (shSigmar1-4) in primary adult cardiac fibroblasts ( $n=3 / g p$ ). A scrambled sequence of shSigmar1-1 was used as a control. (E) Western blot showing the expression level of Sigmar1 upon delivery of 4 specific shRNAs (shSigmar1-4) in primary adult cardiac fibroblasts, using the scrambled sequence of shSigmar1-1 as a control. Tubulin is used as loading control. (F) Representative images of $\alpha$ SMA staining (red) in cardiac fibroblasts upon Sigmar1 silencing using the 4 shRNAs and treatment with TGF- $\beta$, haloperidol, or their combination. Nuclei are stained blue with Hoechst. Scale bar: $100 \mu \mathrm{m}$. (C) Quantification of $\alpha$ SMA mean intensity in cardiac fibroblasts upon Sigmar1 silencing using the 4 shRNAs and treatment with TGF- $\beta$, haloperidol, or their combination $(n=3 / g p)$. Values in $\mathbf{A}, \mathbf{D}$, and $\mathbf{G}$ are mean \pm SEM. ${ }^{*} P<0.05,{ }^{* *} P<0.01,{ }^{* *} P<0.001$ (relative to control in J) by unpaired $t$ test with Welch's correction.

Thus, we tested whether haloperidol, like thapsigargin, was able to induce ER stress in primary fibroblasts. First, we observed that haloperidol upregulated the ER chaperone calreticulin, a marker of ER stress (45), although to a lesser extent than either thapsigargin or tunicamycin (Figure 5, A and B). Next, we determined the expression levels of additional ER stress-related genes, again using thapsigargin and tunicamycin as positive controls. Haloperidol upregulated the expression of Atf3, Atf4, Fkbp11, and Ppp1r15a/Gadd34 at the mRNA level (Supplemental Figure 9A). Ddit3/Chop mRNA was not upregulated at the initial dose tested $(3 \mu \mathrm{M})$, but it increased at a higher concentration $(6 \mu \mathrm{M}$, 
A
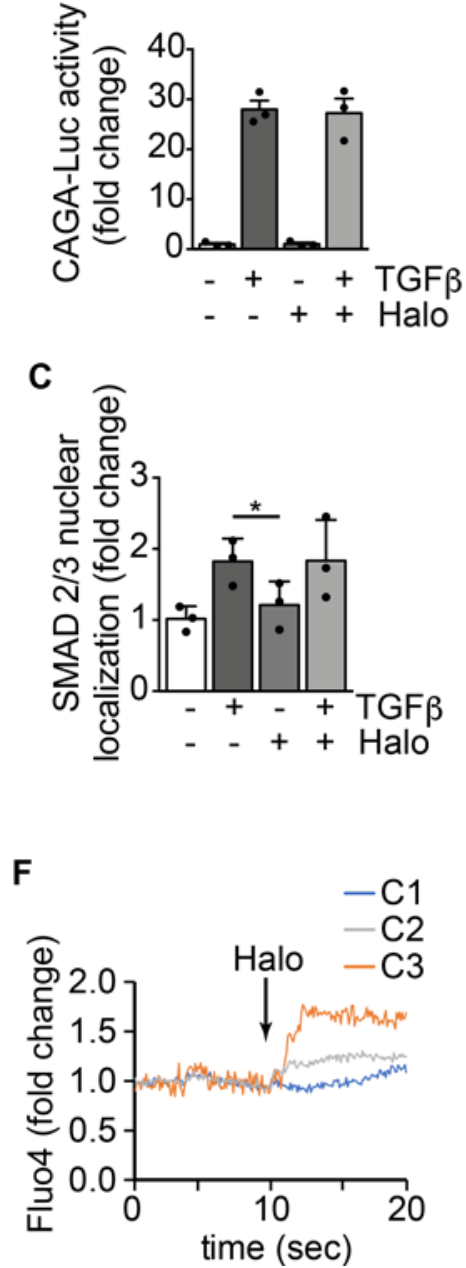

B
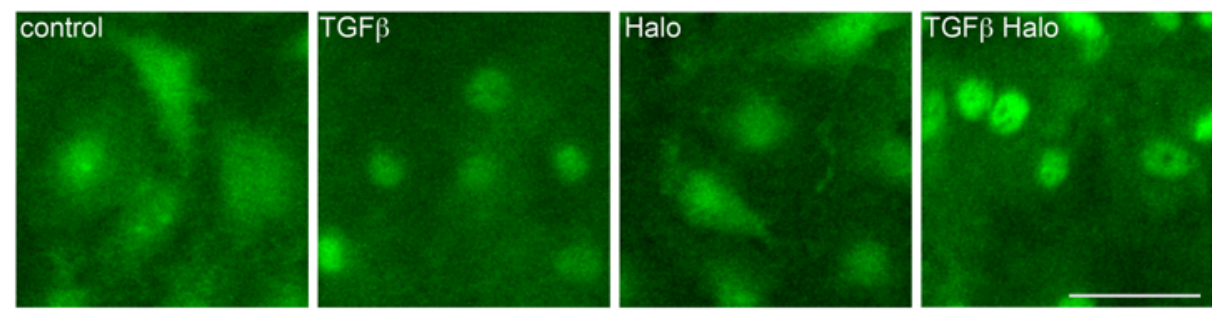

D
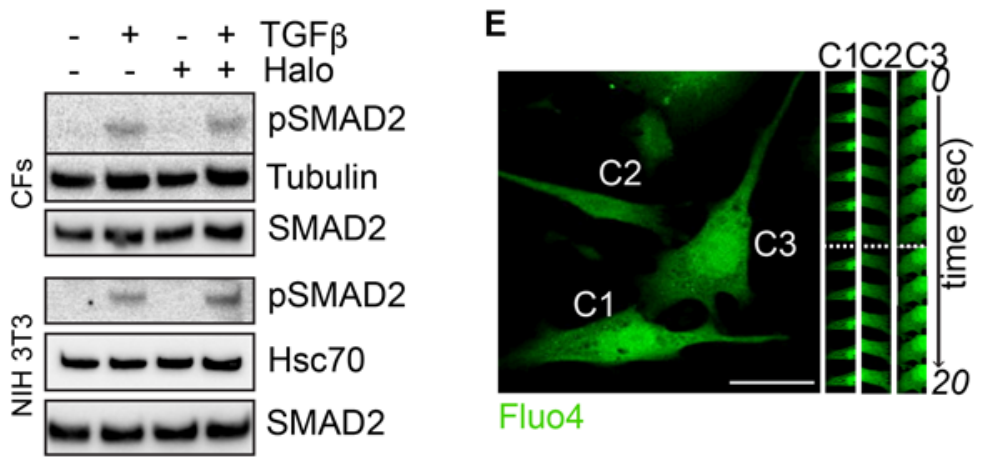

Fluo4

H

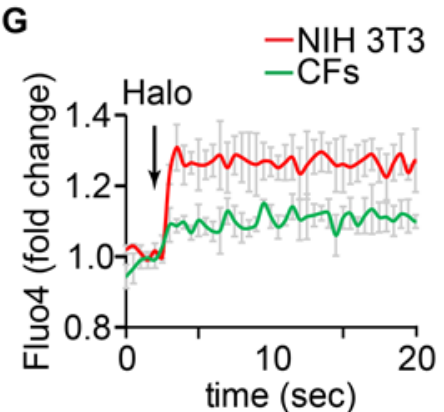

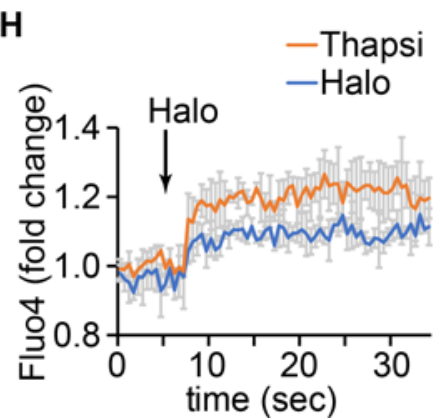

Figure 4. Modulation of $\alpha$ SMA expression by haloperidol involves an increase in intracellular calcium. (A) Luciferase activity of TCF- $\beta$-responsive CACA-Luc reporter upon treatment with TCF- $\beta$, haloperidol, or their combination for 48 hours ( $n=3 / \mathrm{gp}$ ). (B) Representative images of SMAD $2 / 3$ immunofluorescence in primary cardiac fibroblasts treated with TGF- $\beta$, haloperidol, or their combination. (C) Quantification of the nuclear/cytosolic localization of SMAD2/3 in cardiac fibroblasts treated with TCF- $\beta$, haloperidol, or their combination ( $n=3 / g p$ ). (D) Western blot showing the expression level of the phosphorylated form of SMAD2 and loading controls (tubulin and Hsc70) upon treatment with TCF- $\beta$, haloperidol, or their combination in primary cardiac fibroblasts (CFs) and NIH3T3 cells. Total amount of SMAD2 is shown from a blot run in parallel. (E) Representative images of cardiac fibroblasts loaded with the calcium sensitive dye Fluo4. Time-lapse images of specific regions of interest (ROIs) in 3 different cells (C1, C2, and C3) are shown on the right. The dashed line indicates the time of haloperidol treatment. (F) Quantification of mean fluorescence intensity in each ROI (C1 in blue, C2 in gray, and C3 in orange) upon addition of haloperidol at the time indicated by the arrow. (G) Quantification of Fluo4 fluorescence intensity in NIH3T3 cells (red line) and primary cardiac fibroblasts (CFs, green line) over time. Addition of haloperidol is indicated by the arrow. (H) Quantification of whole-cell Fluo4 fluorescence intensity in primary cardiac fibroblasts treated with either haloperidol (blue line) or thapsigargin (orange line), at the time indicated by the arrow. Scale bars in $\mathbf{B}$ and $\mathbf{E}: 50 \mu \mathrm{m}$. Values in $\mathbf{A}$ and $\mathbf{C}$ are mean \pm SEM. ${ }^{*} P<0.05$ by unpaired $t$ test.

Supplemental Figure 9B). A similar ER stress response was induced by haloperidol in primary lung fibroblasts (Supplemental Figure 9C). We also checked the protein levels of PKR-like ER kinase (Perk) and found a substantial increase upon treatment with haloperidol (Figure 5C), in line with previous reports showing the same response in other cell types (46). To confirm the activation of the ER stress pathway, we assessed the levels of phosphorylated forms of both PERK and the eukaryotic translation initiation factor $2 \mathrm{~A}(\mathrm{eIF} 2 \alpha)$ early after haloperidol administration. As shown in Figure 5, D and E, haloperidol increased the phosphorylation of both ER stress mediators. Finally, we demonstrated that PERK is necessary for haloperidol activity by its silencing using a specific siRNA pool, able to significantly decrease PERK protein levels. Haloperidol failed to downregulate $\alpha$ SMA in PERK-silenced fibroblasts (Figure 5, F and G). Overall, these data indicate that haloperidol induces a mild ER stress response in fibroblasts and that PERK is necessary for this effect. 
A

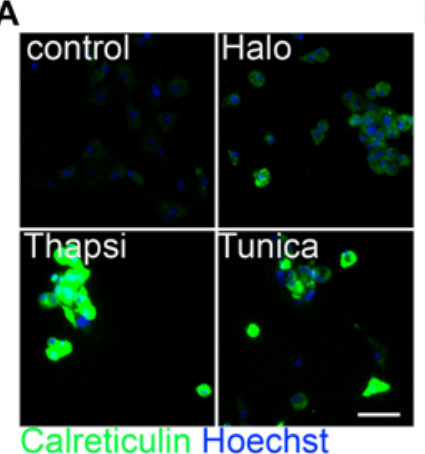

B

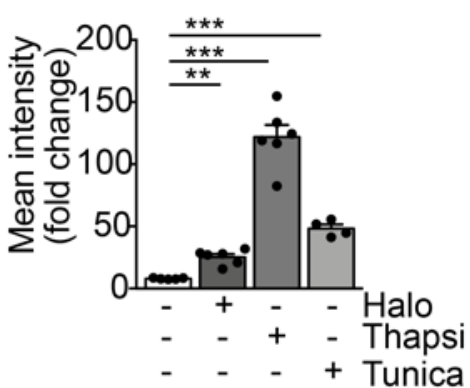

C

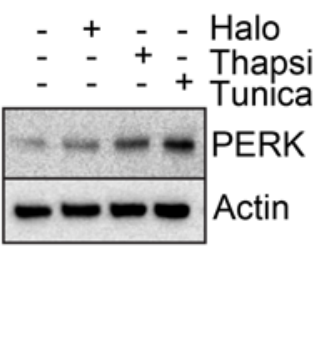

D

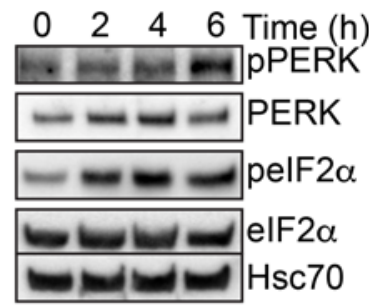

E

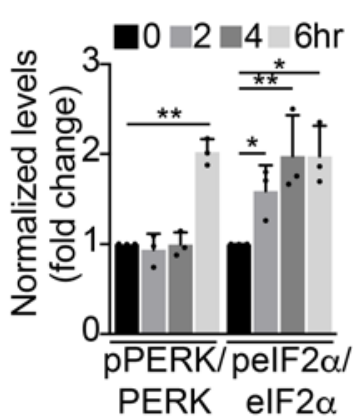

$F$

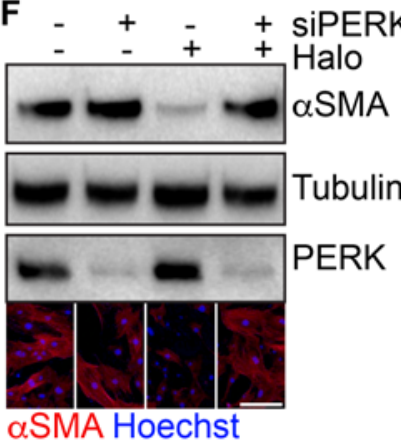

G

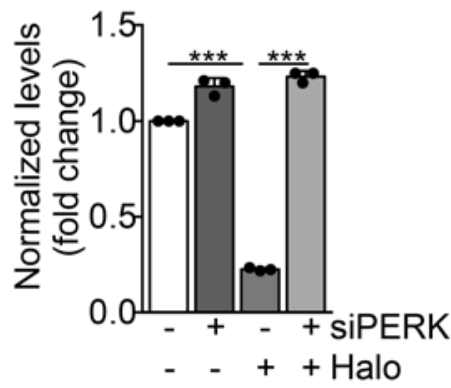

K

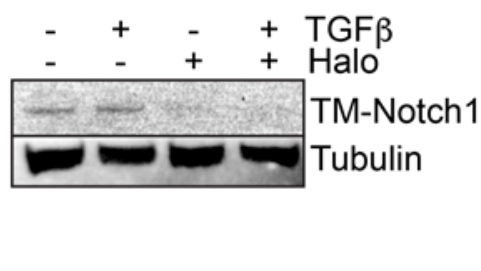

H

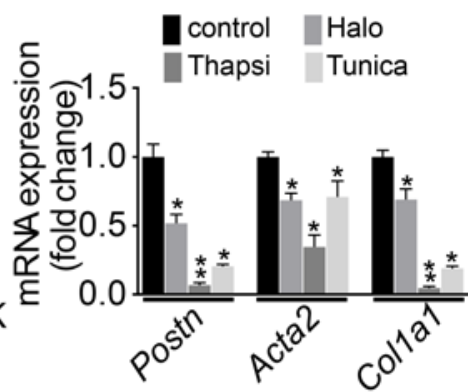

L

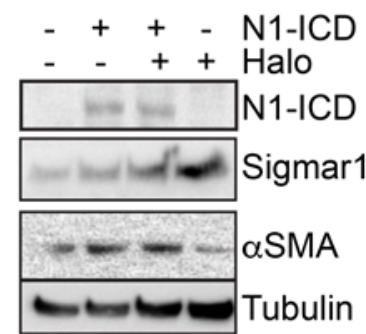

Figure 5. Modulation of ER stress and Notch pathway by haloperidol. (A) Representative images of cardiac fibroblasts stained for calreticulin (green) and treated with haloperidol (Halo), thapsigargin (Thapsi) and tunicamycin (Tunica). Nuclei are stained blue with Hoechst. (B) Quantification of cytosolic calreticulin ( $n=5 / g p)$. (C) Western blot for PERK in cardiac fibroblasts treated with haloperidol, thapsigargin, or tunicamycin for 48 hours. Loading control: actin (D) Western blot for PERK, elF2 $\alpha$, and their phosphorylated forms (p-PERK and p-elF2 $\alpha$ ) in cardiac fibroblasts at the indicated time points after treatment with haloperidol. Hsc70: additional loading control. The 3 blots were run in parallel. (E) Quantification of p-PERK/PERK and p-elF2 $\alpha /$ elF2 $\alpha$ ratios in cardiac fibroblasts at the indicated time points after treatment with haloperidol $(n=3 / \mathrm{gp})$. (F) Western blot for $\alpha \mathrm{SMA}$ and PERK in cardiac fibroblasts treated with haloperidol, siPERK, or their combination. Loading control: tubulin. The 3 blots were run in parallel. Lower panels show representative immunofluorescence for aSMA (red). Nuclei are stained blue with Hoechst. (G) Quantification of $\alpha$ SMA levels in cardiac fibroblasts treated with siPERK, haloperidol, or their combination ( $n=3 / \mathrm{gp}$ ). (H) Levels of mRNA expression of Postn, Acta2, and Col1a1 after treatment with haloperidol, thapsigargin, or tunicamycin ( $n=3$ / gp). (I) Western blot for aSMA upon treatment of cardiac fibroblasts with haloperidol, thapsigargin, or tunicamycin. Loading control: actin. (J) Western blot for the transmembrane form of Notch1 (TM-Notch1), SMAD2 and its phosphorylated form (p-SMAD2) in cardiac fibroblasts treated with either haloperidol or thapsigargin. Loading control: tubulin. The 3 blots were run in parallel. (K) Western blot for TM-Notch1 upon treatment of cardiac fibroblasts with TGF- $\beta$, haloperidol, or their combination. Loading control: tubulin. (L) Western blot for the intracellular form of Notch1 (Notch1-ICD), $\alpha$ SMA, and Sigmar1 upon transfection of a constitutively active form of Notch1 $\left(\mathrm{N} 1^{1 \mathrm{CD}}\right)$ either alone or in combination with haloperidol. Loading control: tubulin. The 3 blots were run in parallel. Scale bars in $\mathbf{A}$ and $\mathbf{H}: 50 \mu \mathrm{m}$. Values in B, E, G, and $\mathbf{H}$ are mean $\pm \mathrm{SEM}$. ${ }^{*} P<0.05,{ }^{* *} P<0.01,{ }^{* *} P<0.001$ by unpaired $t$ test.

Is this relevant to the observed effect of haloperidol in reducing myofibroblast activation and fibrosis? Interestingly, the 2 potent inducers of ER stress, thapsigargin and tunicamycin, were even more potent than haloperidol in reducing the expression of 3 major markers of myofibroblast activation, Postn, Acta2, and Colla1, in primary cardiac and lung fibroblasts (Figure 5, H and I, and Supplemental Figure 9C). These results are in line with publicly available microarray data sets, in which mouse embryonic fibroblasts derived from different strains were treated with tunicamycin for either 4 or 8 hours. As shown in Supplemental Figure 9, D and E, all data sets invariably showed increased expression of the ER stress-induced genes Atf3, Atf4, and Fkbp11, and a tendency toward downregulation of the profibrotic genes Postn, Acta2, and Colla1. 
The ER stress response is known to lead to the accumulation of unfolded proteins within the ER (47). As thapsigargin has been recently shown to interfere with the folding and expression of Notch1 in cancer cells (48-51) and Notch1 in turn seems to positively regulate the synthesis of aSMA in multiple cell types (52-54), we checked whether the same holds true in primary cardiac fibroblasts. We found that both haloperidol and thapsigargin potently reduced the levels of transmembrane Notch1 (TM-Notch1), without interfering with the canonical TGF- $\beta$ pathway, as indicated by the unchanged levels of Smad2 phosphorylation (Figure 5J). The inhibitory effect of haloperidol on TM-Notch1 expression was still evident when haloperidol was administered in combination with TGF- $\beta$ (Figure 5K). The Notch target genes Hes 1, Hey1, Cond1, and Notch1 itself were also downregulated by haloperidol, although to a variable extent, in both cardiac and lung primary fibroblasts (Supplemental Figure 10A). Consistently, treatment of cardiac fibroblasts with the Notch inhibitor DAPT resulted in a dose-dependent reduction in the expression of $\alpha$ SMA, similar to what we obtained by treating the same cells with the TGF- $\beta$ inhibitor SB43152 (Supplemental Figure 10B). To understand whether Notch1 processing was affected by haloperidol, we investigated its intracellular localization and found that upon treatment with haloperidol Notch 1 was retained within the ER compartment, as shown by its colocalization with the ER marker calreticulin (Supplemental Figure 11, A-E).

To further demonstrate that the effect of haloperidol on $\alpha$ SMA expression was mediated by the downregulation of Notch signaling, we overexpressed the constitutively active intracellular domain of Notch1 (Notch1-ICD) in NIH3T3 fibroblasts and found that it upregulated the expression of $\alpha$ SMA. Moreover, its overexpression in combination with haloperidol almost totally inhibited the effect of the drug, restoring the levels of $\alpha$ SMA and reducing the levels of Sigmar1 (Figure 5L).

Haloperidol inhibits aSMA expression by myofibroblasts in vivo. We then investigated whether the effect of haloperidol in inhibiting myofibroblast conversion of fibroblasts was also exerted in 3 different in vivo models of fibrosis, namely cardiac fibrosis induced by left anterior descending coronary artery (LAD) ligation, bleomycin-induced lung fibrosis, and cancer-associated fibrosis in a model of orthotopic lung cancer.

First, we ligated the LAD to generate an acute myocardial infarction (MI) in both CD1 and COLL-EGFP mice, which allows reliable visualization of fibroblasts by fluorescence microscopy. As expected, LAD ligation resulted in a large fibrotic scar, populated by $\mathrm{EGFP}^{+}$fibroblasts (Figure 6, A and B). Treatment with haloperidol for 10 days starting at the time of $\mathrm{MI}$ resulted in a significant reduction in scar size (Figure $6, \mathrm{~B}$ and $\mathrm{C}$ ) as well as in the number of $\alpha \mathrm{SMA}^{+}$myofibroblasts (Figure $6, \mathrm{D}$ and $\mathrm{E}$ ), without any change in the density of fibroblasts within the scar region (Figure $6 \mathrm{~F}$ ). However, when we monitored heart function over a period of 8 weeks after MI, we found that all the major echocardiography parameters, including ejection fraction (EF), fractional shortening (FS), end-diastolic left ventricular volume (EDLV), and end-systolic left ventricular volume (ESLV) were not significantly changed by haloperidol treatment (Figure 6, G-J). In accordance, the early effect on scar size was not evident at 8 weeks, which was possibly related to the previously described cardiotoxic effect of haloperidol (55) (Supplemental Figure 12, A and B).

Next, we tested the effect of haloperidol in a model of pulmonary fibrosis (Figure 7A). As shown in Figure 7, B-D, the intratracheal administration of bleomycin into COLL-EGFP mice resulted in the formation of several COLL-EGFP ${ }^{+}$fibrotic foci, which were significantly reduced in both number and intensity of collagen expression upon treatment by haloperidol for 10 days. In addition, $\alpha$ SMA positivity in extravascular regions was markedly reduced by haloperidol in the same lung sections (Figure 7, E-G). We performed LC-MS/MS analysis to confirm the availability of haloperidol in tissues using an established protocol $(56,57)$ and found that it distributed preferentially to the lung compared with the heart, independent of the treatment with bleomycin, reaching a concentration that was one-tenth of that measured in cultured fibroblasts (Supplemental Figure 13).

We also compared the effect of haloperidol to that exerted by the 2 antifibrotic drugs approved for use in IPF patients, nintedanib and pirfenidone. As shown in Supplemental Figure 14, A-C, all 3 drugs significantly inhibited $\alpha$ SMA expression in extravascular regions compared with lungs injected with bleomycin only. While pirfenidone was the most potent drug in reducing myofibroblast activation in this model, no significant differences were observed between haloperidol and nintedanib. Moreover, we compared the capacity of the 3 drugs to reduce $\alpha \mathrm{SMA}$ expression in isolated lung fibroblasts at both mRNA and protein levels and observed that haloperidol was the most effective one in both cases (Supplemental Figure 14, D-F).

To assess the therapeutic potential of haloperidol to reverse fibrosis, we started the treatment 10 days after the induction of fibrosis in the same model. Again, haloperidol was effective in reducing aSMA levels in lung fibroblasts (Supplemental Figure 15, A-C). In this therapeutic trial, haloperidol was as effective as pirfenidone 
A

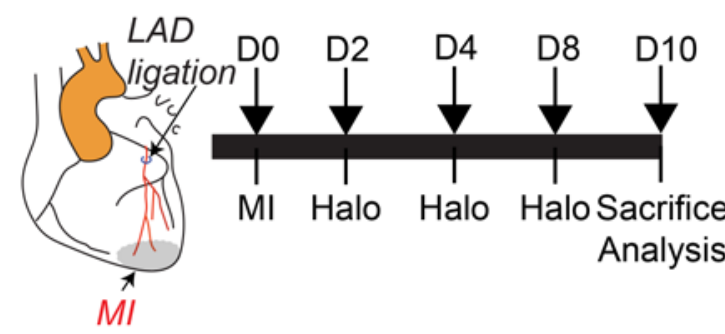

B

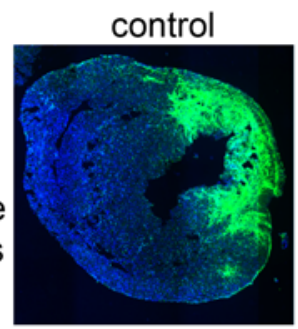

C

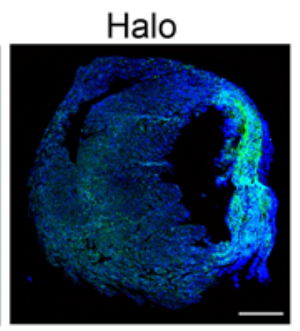

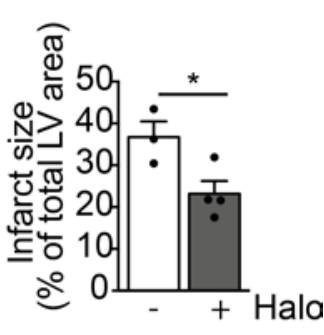

COLL-EGFP Hoechst

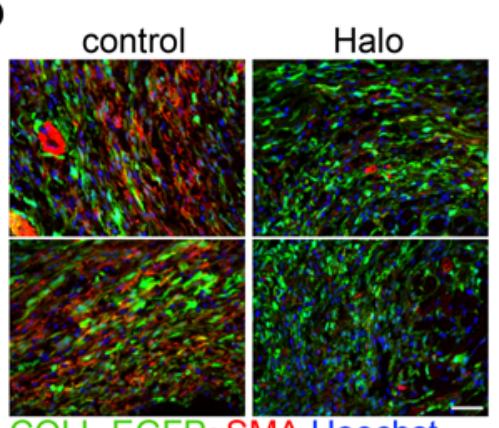

COLL-EGFPaSMA Hoechst

H

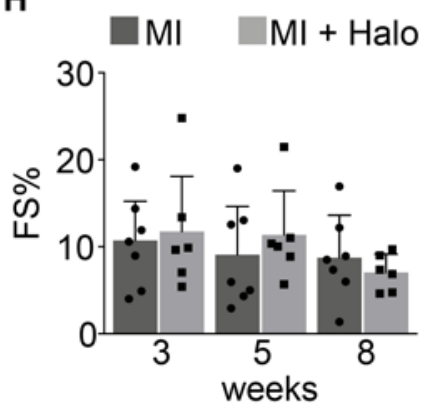

E

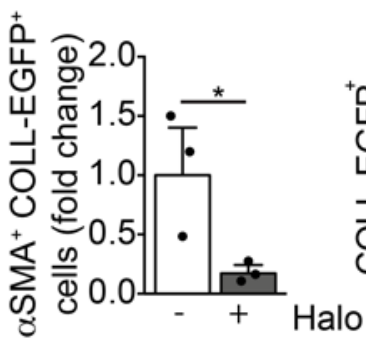

$\mathbf{F}$

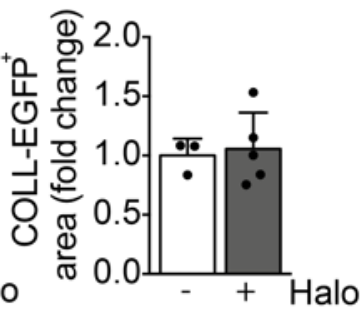

G

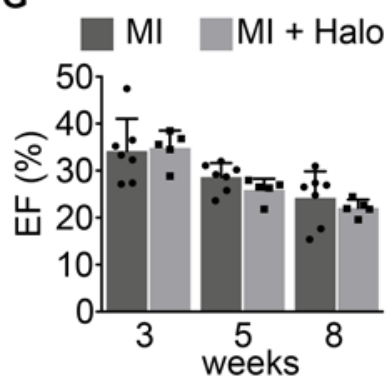

Figure 6. Haloperidol inhibits $\alpha$ SMA expression by cardiac myofibroblasts in vivo. (A) Schematic of the cardiac fibrosis following myocardial infarction (MI) induced by the ligation of the left descendent anterior coronary artery (LAD). The aorta is indicated in orange, the LAD in red, and the MI in gray. (B) Representative images of the heart sections of COLL-EGFP mice at 10 days after MI, treated with either PBS (control) or haloperidol (Halo). Collagen expression is shown in green (COLL-EGFP) and nuclei are stained blue with Hoechst. (C) Quantification of infarct size in mice treated with either PBS or haloperidol on day 10 after MI ( $n>3 / g p)$. (D) Representative images of heart sections of COLL-EGFP mice, following MI and treatment with either PBS or

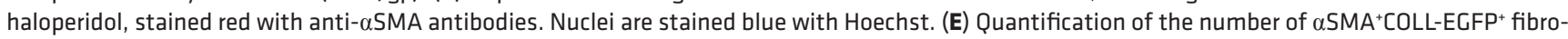
blasts in infarcted hearts treated with either PBS or haloperidol ( $n>3 / \mathrm{gp})$. (F) Quantification of the COLL-EGFP+ area in infarcted hearts treated with either PBS or haloperidol ( $n>3 / \mathrm{gp}$ ). (G) Quantification of the ejection fraction (EF) in infarcted mice treated with either PBS or haloperidol at 3, 5, and 8 weeks after MI ( $n>5 / \mathrm{gp})$. (H) Quantification of the fractional shortening (FS) in mice subjected to MI and treated with either PBS (black bars) or haloperidol (gray bars) at 3, 5, and 8 weeks after MI ( $n>5 / \mathrm{gp}$ ). (I) Quantification of the end-diastolic left ventricular volume (EDLV) in mice subjected to MI and treated with either PBS (black bars) or haloperidol (gray bars) at 3, 5, and 8 weeks after MI ( $n>5 / g p)$. (J) Quantification of the end-systolic left ventricular volume (ESLV) in mice subjected to MI and treated with either PBS (black bars) or haloperidol (gray bars) at 3, 5, and 8 weeks after MI ( $n>5 / g p$ ). Scale bars: 1 mm (B) and $100 \mu \mathrm{m}$ (D). Values in $\mathbf{C}$ and $\mathbf{E}-\mathbf{J}$ are mean \pm SEM. ${ }^{*} P<0.05$ by unpaired $t$ test.

and always superior to nintedanib. To confirm the induction of ER stress also in vivo, we stained the lung sections with antibodies specifically recognizing the active, phosphorylated form of PERK. While p-PERK was abundantly expressed by other cell types, most likely alveolar epithelial cells in the fibrotic lung, haloperidol treatment resulted in the specific increase of p-PERK in myofibroblasts (Supplemental Figure 16).

Finally, we tested whether haloperidol was able to regulate $\alpha$ SMA expression in CAFs in a model of lung cancer (Figure 8A). We injected a bolus of LG1233 lung cancer cells derived from $\mathrm{Kras}^{\mathrm{G} 12 \mathrm{D} /+} \operatorname{Trp}^{53^{-/-}}$mice, herein referred to as LG cells, into the tail vein of syngeneic C57BL/6 mice. This resulted in the formation of multiple tumor nodules spread throughout the lung parenchyma (Figure 8B), in which cancer cells were interspersed with CAFs, including vimentin ${ }^{+}$fibroblasts and $\alpha \mathrm{SMA}^{+}$myofibroblasts (Supplemental Figure 17). Administration of haloperidol for 10 days resulted in a remarkable reduction in the number of tumor nodules, 
A

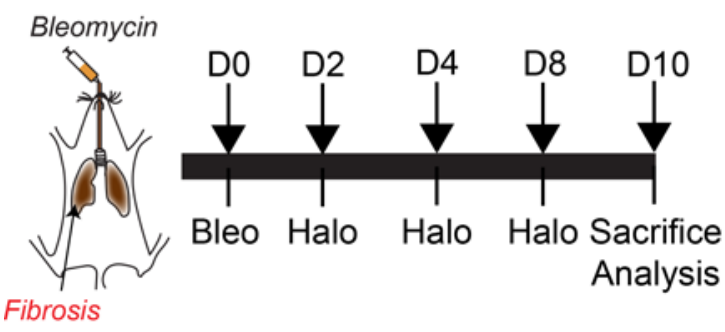

B

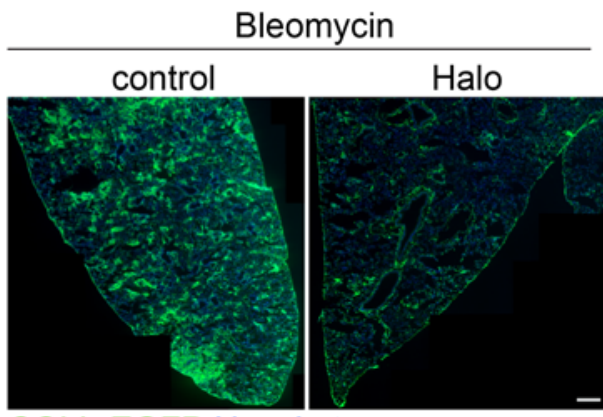

C

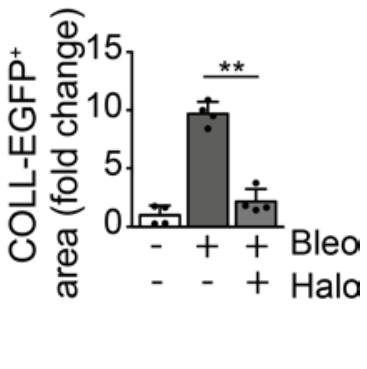

D

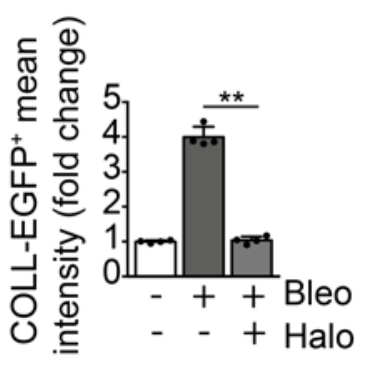

E

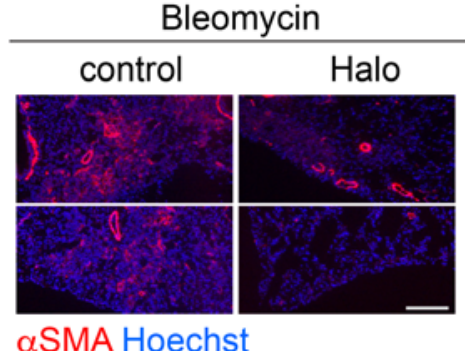

$\mathbf{F}$

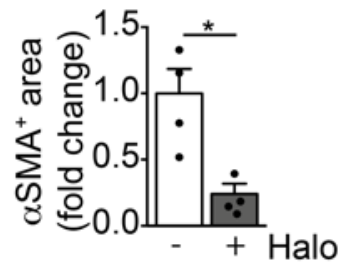

G

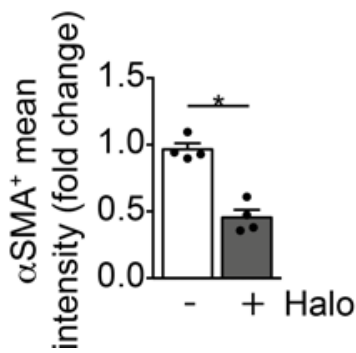

Figure 7. Haloperidol inhibits aSMA expression by lung myofibroblasts in vivo. (A) Schematic of lung fibrosis induced by intratracheal injection of bleomycin (orange). (B) Representative images of lung sections of COLL-EGFP mice exposed to bleomycin and treated with either PBS (control) or haloperidol. Nuclei are stained blue with Hoechst. (C) Quantification of the COLL-EGFP+ area in lungs exposed to bleomycin upon treatment with either PBS or haloperidol ( $n=4 / g p)$. (D) Quantification of the mean cell intensity of COLL-EGFP+ in lung fibroblasts exposed to bleomycin upon treatment with either PBS or haloperidol ( $n=4 / \mathrm{gp}$ ). (E) Representative images of lung sections from mice exposed to bleomycin upon treatment with either PBS (control) or haloperidol, stained red with anti- $\alpha$ SMA antibodies. Nuclei are stained blue with Hoechst. (F) Quantification of $\alpha S^{2} A^{+}$area in lungs of mice exposed to bleomycin upon treatment with either PBS or haloperidol ( $n=4 / \mathrm{gp}$ ). (C) Quantification of the mean cell intensity of $\alpha \mathrm{SMA} \mathrm{A}^{+}$in lung fibroblasts exposed to bleomycin upon treatment with either PBS or haloperidol ( $n=4 / \mathrm{gp})$. Scale bars: $1 \mathrm{~mm}(\mathbf{B})$ and $50 \mu \mathrm{m}(\mathbf{E})$. Values in C, D, F, and G are mean \pm SEM.* $P<0.05,{ }^{* *} P<0.01$ by unpaired $t$ test.

as well as in the area occupied by cancer cells (Figure 8, B-E). This anticancer activity was associated with a significant decrease in the expression of aSMA in the nonvascular tumor area (Figure 8, C and F). Moreover, these $\alpha \mathrm{SMA}^{+}$cells almost stopped proliferating in response to haloperidol (Figure $8, \mathrm{G}$ and $\mathrm{H}$ ), without showing signs of either cellular senescence or apoptosis in profibrotic conditions (Supplemental Figure 18).

Collectively, these results indicate that haloperidol effectively inhibits activation of myofibroblasts, likely reverting them back to a quiescent phenotype in 3 different models of fibrosis in vivo.

Haloperidol inhibits human myofibroblast activation. As a step toward a possible use of haloperidol as a novel antifibrotic agent in humans, we investigated its effect on primary fibroblasts derived from human skin. First, we confirmed that haloperidol reduced the levels of aSMA in both basal conditions and upon treatment with TGF- $\beta$ (Figure 9, A and B). Second, we performed live imaging experiments and again found that haloperidol increased Fluo4 fluorescence, indicating increased levels of intracellular calcium (Figure 9, C and D, and Supplemental Video 2). Finally, we observed that the downregulation of $\alpha \mathrm{SMA}$ expression, either in the absence or in the presence of TGF- $\beta$, was invariably associated with reduced levels of TM-Notch1 (Figure 9E).

Collectively, these results imply that haloperidol effectively inhibits the activation of fibroblasts derived from various organs and species, through a mechanism that involves binding to Sigmar1, an increase in the intracellular calcium levels, mild ER stress, and inhibition of the Notch pathway, as schematically depicted in Figure 9F.

\section{Discussion}

Using an innovative approach based on the availability of transgenic animals expressing RFP under the control of the aSMA promoter, we have performed HTS and identified several drugs affecting the differentiation of primary fibroblasts into myofibroblasts. Among the top 10 hits were dexamethasone and prednisone, two corticosteroids that have been previously shown to reduce fibrosis and are used to treat patients 
A

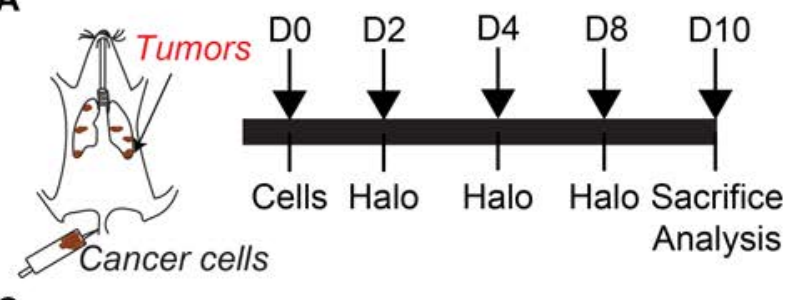

C

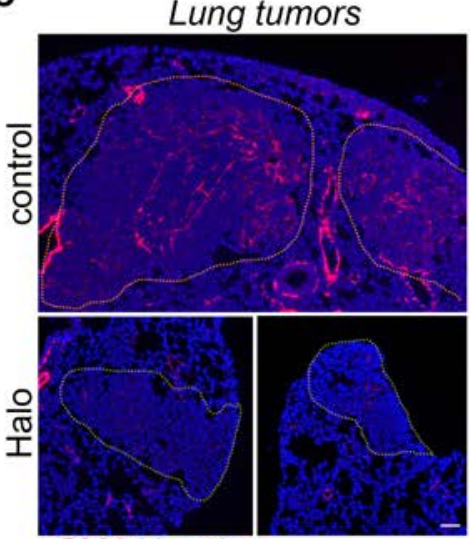

aSMA Hoechst

G

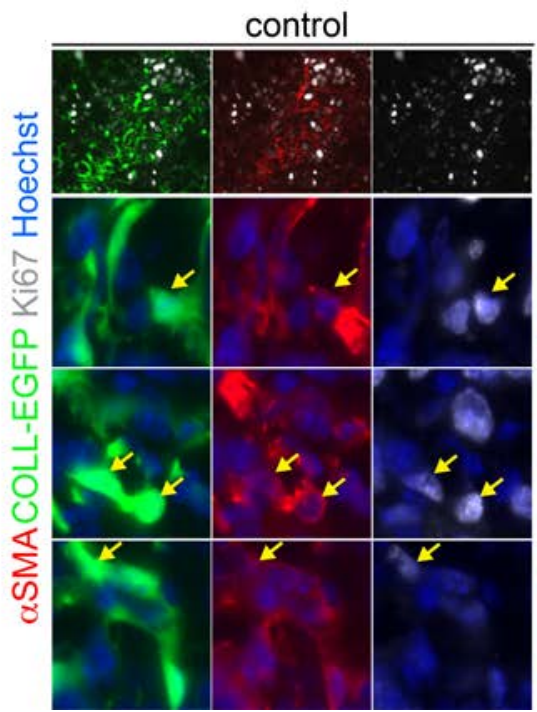

B
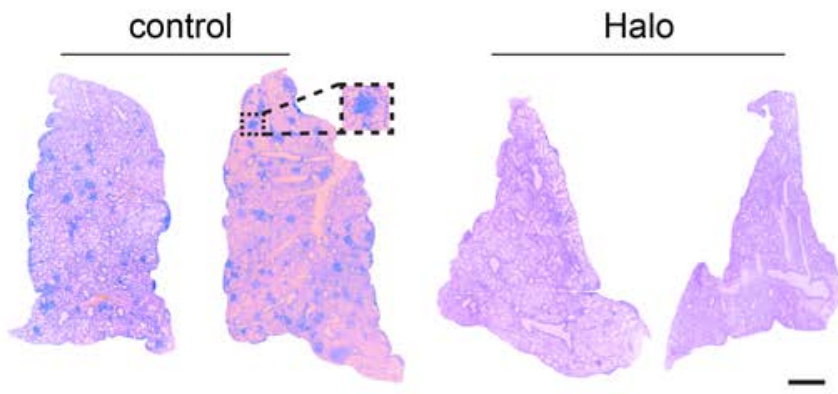

D

E
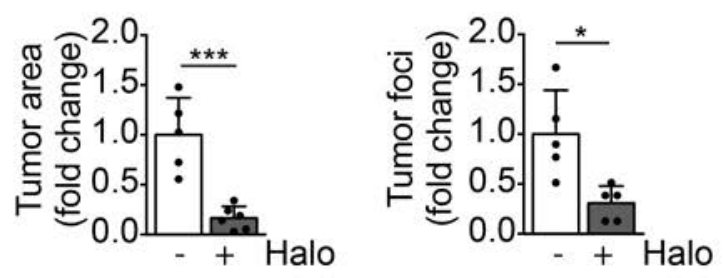

F

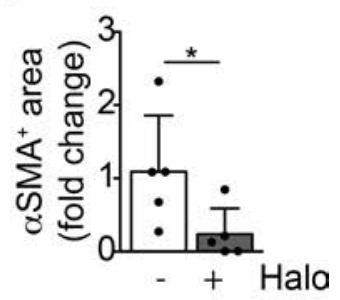

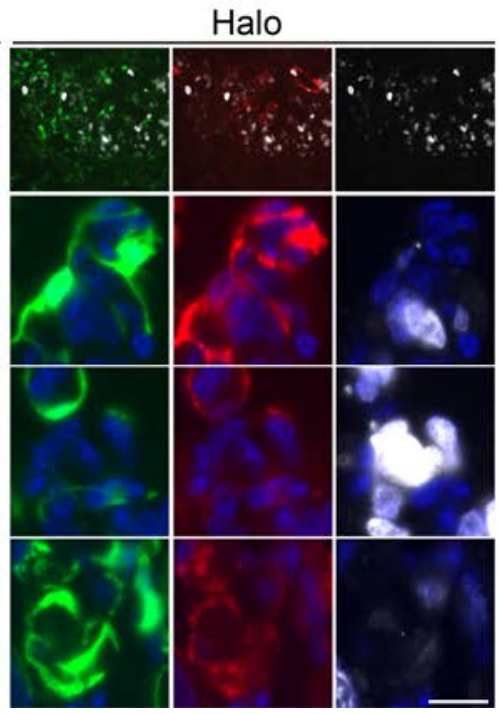

H

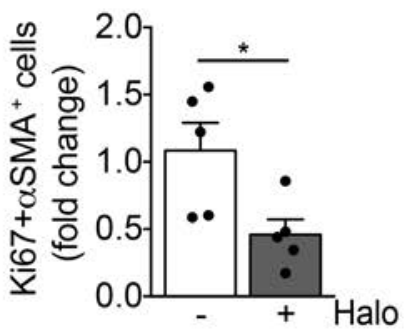

Figure 8. Haloperidol inhibits $\alpha$ SMA expression by cancer-associated myofibroblasts in vivo. (A) Schematic of orthotopic lung tumors associated with cancer-associated fibroblasts (cancer cells are indicated in brown). (B) Hematoxylin and eosin staining of representative sections of lungs of mice injected with LG cancer cells into the tail vein, upon treatment with either PBS (control) or haloperidol. (C) $\alpha$ SMA staining (red) of sections of tumor-bearing lungs in mice treated with either PBS (control) or haloperidol. Nuclei are stained blue with Hoechst. (D) Quantification of the tumor area in lungs of mice treated with either PBS or haloperidol ( $n=5 / \mathrm{gP}$ ). (E) Quantification of the number of tumor foci in lungs of mice treated with either PBS or haloperidol ( $n=5 / \mathrm{gp}$ ). (F) Quantification of $\alpha \mathrm{SMA}^{+}$area in tumor-bearing lungs of mice upon treatment with either PBS or haloperidol $(n=5 / \mathrm{gp})$. (G) Representative images of $\mathrm{Ki}-67$ (white) and $\alpha$ SMA (red) staining in sections of lung in COLL-EGFP mice (fibroblasts in green) treated with PBS (control) or haloperidol. Arrows indicate Ki-67+COLL-EGFP+ $\alpha S M A^{+}$cells. (H) Quantification of the Ki-67+ $\alpha S M A^{+}$myofibroblasts in lung cancer sections of COLL-EGFP mice treated with either PBS or haloperidol $(n=5 / \mathrm{gp})$. Scale bars: $1 \mathrm{~mm}$ (B) and $50 \mu \mathrm{m}(\mathbf{C}$ and $\mathbf{~})$. Values in $\mathbf{D}-\mathbf{F}$ and $\mathbf{H}$ are mean $\pm \mathrm{SEM} .{ }^{*} P<0.05$, ${ }^{* * *} P<0.001$ by unpaired $t$ test.

with IPF (14), but are fraught with major side effects, including bone loss, diabetes, and hypertension (58). The second hit was haloperidol, a potent antipsychotic drug, which has been successfully used to treat psychosis, agitated states, and delirium for over half of a century. A few studies have revealed antiinflammatory properties of haloperidol (59), resulting in reduced lung fibrosis, without any further mechanistic explanation of this in vivo effect (60). Here, we show that haloperidol inhibits the activation of myofibroblasts in different organs, by binding to Sigmar1. This in turn activates an intracellular signaling cascade, 
A

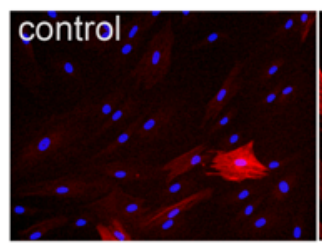

aSMA Hoechst
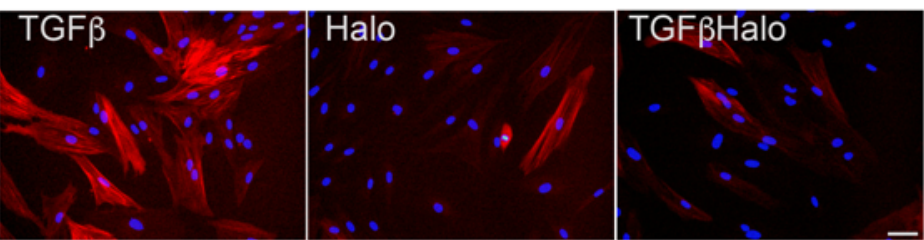

B

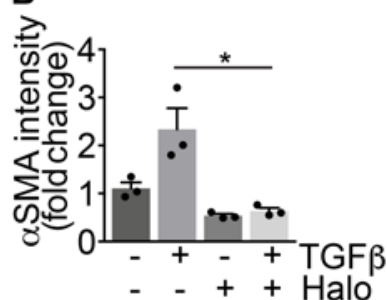

C

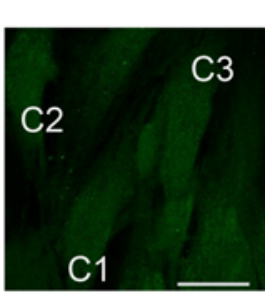

Fluo4
D \&

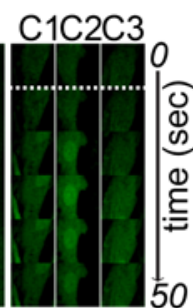

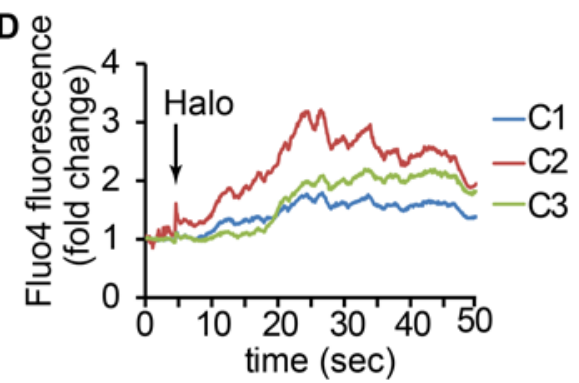

E

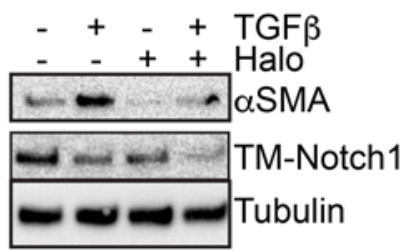

$\mathbf{F}$

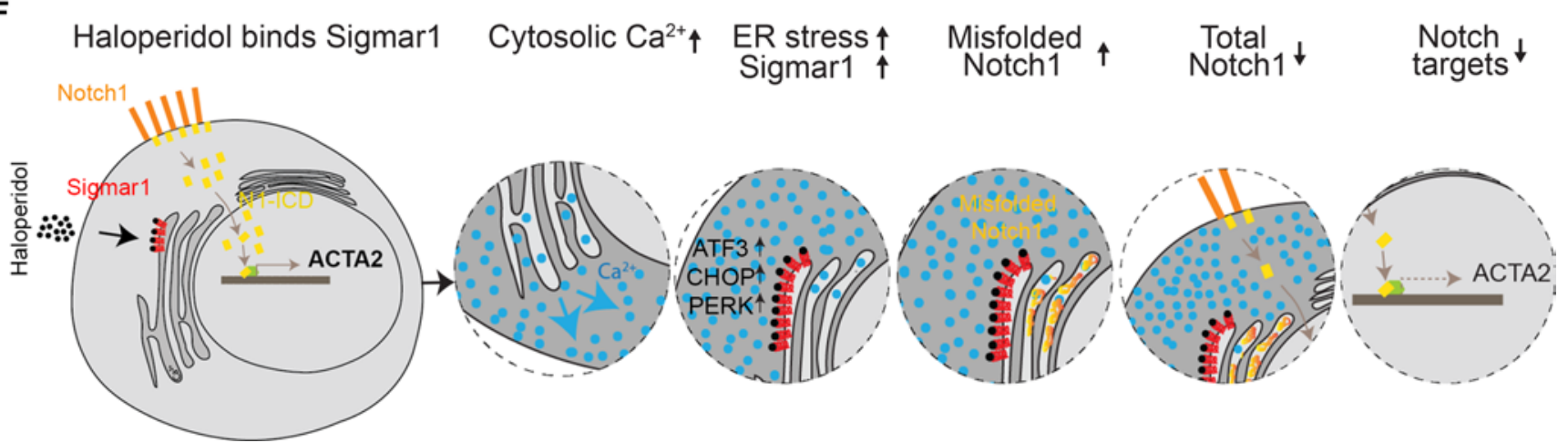

Figure 9. Haloperidol inhibits human myofibroblast activation. (A) Adult human dermal fibroblasts treated with TGF- $\beta$, haloperidol, or their combination and stained with anti- $\alpha$ SMA antibodies (red). Nuclei are stained blue with Hoechst. (B) Quantification of the $\alpha$ SMA mean fluorescence intensity upon treatment with TGF- $\beta$, haloperidol, or their combination $(n=3 / \mathrm{gp})$. Values are mean \pm SEM. ${ }^{*} P<0.05$ by unpaired $t$ test. (C) Representative images of human fibroblasts loaded with the calcium-sensitive dye Fluo4 and treated with haloperidol at the time indicated by the dashed line. Time-lapse images of specific regions of interest (ROIs) in 3 different cells (C1, C2, and C3) are shown on the right. (D) Quantification of mean fluorescence intensity in each ROI (C1 in blue, $\mathrm{C} 2$ in red, and C3 in green) upon addition of haloperidol at the time indicated by the arrow. (E) Western blot showing the expression of TM-Notch1 and $\alpha$ SMA after treatment with TGF- $\beta$, haloperidol, or their combination. Tubulin is used as loading control. Blot for $\alpha$ SMA was run in parallel. (F) Schematic of a proposed model for the mechanism of action of haloperidol in regulating the expression of $\alpha$ SMA in fibroblasts. Scale bars in $\mathbf{A}$ and $\mathbf{C}$ : $50 \mu \mathrm{m}$.

which leads to an increase in the cytoplasmic calcium levels, the activation of mild ER stress response, reduced Notch1 signaling, and eventually reduced expression of profibrotic genes.

In the heart, this antifibrotic mechanism resulted in a transient reduction of infarct size at 10 days, consistent with a previous report showing reduced incidence of MI at autopsy in psychiatric patients treated with haloperidol (61). However, this beneficial effect was lost at later time points, which was possibly related to the toxic effect of haloperidol on ATP production and contractility in cardiomyocytes (55), as also documented by the occurrence of heart arrhythmias, prolongation of QT interval, and torsade de pointes in patients treated with haloperidol $(62,63)$.

In the lung, we observed a potent effect of haloperidol in reducing myofibroblast activation in a model of bleomycin-induced fibrosis. Although pirfenidone was slightly more effective than haloperidol in the protocol tested for prevention, they both show the same therapeutic activity when administered once the fibrotic process was already in place. In addition, when we assessed $\alpha$ SMA expression in lung fibroblasts, haloperidol was consistently the most effective drug.

We also demonstrated the efficacy of haloperidol in an orthotopic lung cancer model. Haloperidol is commonly used in cancer patients for the control of nausea, vomiting, and agitation $(64,65)$. In our model, we observed a clear reduction in both the tumor area and the number of tumor foci, associated with a decreased 
number of intratumoral myofibroblasts. Haloperidol was previously shown to inhibit tumor growth, possibly by binding to Sigmar1 expressed by various types of cancer cells $(41,66)$, and repurposing of haloperidol as an anticancer drug has been proposed for the treatment of glioblastoma (67). As we did not detect any inhibitory effect of haloperidol on the proliferation of LG cells (data not shown), we believe that in our model the main mechanism by which it has reduced tumor growth in vivo was through the inhibition of myofibroblast activation. Thus, haloperidol could be used as a pleiotropic drug, modulating the growth of cancers expressing Sigmar1.

The biology of Sigmar1 is very complex and to a large extent still poorly defined. It is a single $25-\mathrm{kDa}$ polypeptide and a chaperone protein immersed in lipid rafts of the ER, where it interacts with mitochondria and the mitochondria-associated ER membrane domain (MAM). Upon activation, Sigmar1 modulates the function of multiple receptors and ion channels, contributing to cellular calcium homeostasis (68). Our docking model shows that haloperidol in its highest-ranked binding pose interacts with all residues in the binding pocket of Sigmar1, including Phe133, Glu172, Leu105, Met93, Leu95, Ile178, Val84, His154, and Val152, in line with previous studies pointing to Glu172 as an essential residue for Sigmar1-haloperidol binding (69, 70). Interestingly, we found that haloperidol potently upregulates Sigmar1 at the protein level, with no significant changes in its transcription (transcriptional data not shown). This has been previously observed in hepatocellular carcinoma and neuronal cells $(42,71)$, but to our knowledge not reported in fibroblasts so far. Our data suggest that the binding of haloperidol to Sigmar1 might induce the release of calcium from the ER into the cytosol, thereby promoting ER stress, which could in turn upregulate Sigmar1 levels. Consistent with this hypothesis, Sigmar1 upregulation was reported upon cell treatment with other ER stress inducers, such as glucose, heat shock, and thapsigargin (72). Also, previous genetic screenings identified either the noncanonical TGF- $\beta$ pathway or calcium as a major regulator of myofibroblast activation (23-25). For instance, TRPC6-mediated calcium signaling induced myofibroblast differentiation, although this channel essentially controls calcium influx from the extracellular space and not its release from the ER, as in our model (24).

Is the ER stress also mediating the observed downregulation of Notch1 levels in response to haloperidol? Although the definitive answer to this question still needs to be provided, our data support the emerging view that ER stress might indeed control the Notch pathway, which is implicated in multiple processes during both development and disease (73). We observed that fibroblast treatment with haloperidol resulted in the upregulation of various ER stress markers, including Atf3, Fkbp11, and Gadd34, similarly to the potent ER stress inducers thapsigargin and tunicamycin. ER stress is known to potentially interfere with the expression of proteins, which depend on calcium for their proper folding $(74,75)$. Specifically, the Notch1 receptor contains 36 EGF and 3 Lin12/Notch repeats, which all require calcium ions to properly fold (76-78). Indeed, thapsigargin, by altering calcium concentration within the ER, was shown to interfere with Notch1 maturation, resulting in the overall inhibition in Notch1 expression and signaling in leukemia cells (48). We also found that haloperidol and thapsigargin similarly downregulated the levels of the Notch1 transmembrane form in both murine and human primary fibroblasts. This is in line with previous work in flies, showing that misfolded Notch1 in Ero1 mutant cells is retained and not properly exported from the ER to the cell membrane (79). Importantly, the overexpression of the constitutively active Notch1-ICD, lacking EGF and Lin12/ Notch repeats, inhibited the effect of haloperidol and restored $\alpha \mathrm{SMA}$ in fibroblasts. These data are also in line with multiple lines of evidence, supporting an antifibrotic role of the Notch pathway $(80,81)$. Notch pathway components were found upregulated in models of cardiac, lung, skin, and liver fibrosis and chemical inhibition of the pathway invariably reduced the fibrotic signature, improving the outcome of the disease (82-85).

Similarly, the role of ER stress in fibrotic diseases has been extensively investigated, with conflicting results $(86,87)$. ER stress markers, such as Atf4, Chop, and Atf6, appear upregulated in patients with IPF $(88,89)$. In addition, ER stress induced by the accumulation of misfolded protein, such as in the case of transgenic mice expressing a mutant form of the surfactant protein A2 (SFTPA2) in alveolar epithelial cells, also results in lung fibrosis. Although these data tend to indicate that ER stress is detrimental and enhance a profibrotic response, other evidence, including ours, supports opposite conclusions. For instance, induction of ER stress by either thapsigargin or tunicamycin resulted in reduced Colla1 expression by mouse embryonic fibroblasts $(88,90,91)$. Whether these discrepancies might be attributed to a different role of the ER stress in different cell types (i.e., ER stress might promote a fibrotic response in alveolar cells and reduce it in fibroblasts) or the extent of the ER stress response might result in different outcome (i.e., a mild ER stress might be protective, whereas a strong ER stress might be detrimental) still remains an open question.

Overall, our data indicate that haloperidol might be considered a novel drug able to interfere with myofibroblast activation, supporting its repurposing for the treatment of various diseases involving a profibrotic 
response. In addition, we provide mechanistic insights linking Sigmar1, calcium homeostasis, ER stress, and the Notch1-mediated transcription of $\alpha$ SMA in primary fibroblasts derived from different organs. The evidence that this pathway is also active in human fibroblasts could set the stage for the validation of novel therapeutic targets in inhibiting fibrosis.

\section{Methods}

For more methodological details see supplemental material.

Cell culture. Primary murine fibroblasts were isolated by mechanical and enzymatic digestion. Primary human fibroblasts and NIH3T3 were obtained from Lonza and ATCC, respectively. The lung adenocarcinoma cell line LG1233 was derived from lung tumors of C57BL/6 KP mice and was provided by Tyler Jacks (Massachusetts Institute of Technology, Cambridge, Massachusetts, USA) (92). All cells were maintained in Dulbecco's Modified Eagle's Medium (DMEM), supplemented with 10\% FBS. Myofibroblast differentiation was induced by TGF- $\beta$.

HTS. Primary murine cardiac fibroblasts were plated in 384-well plates. The next day, the FDA-approved compound library (640 compounds, ENZO Life Sciences) was spotted on top of the cells at a final concentration of $10 \mu \mathrm{M}$ with $0.1 \%$ DMSO. After an additional 48 hours, the cells were fixed and stained with Hoechst 33342 and HCS Cell Mask Deep Red staining. Image acquisition was performed using the ImageXpress Micro HC screening microscope to analyze aSMA expression (TRITC channel, cellular mean fluorescence intensity) using MetaXpress software. Compounds exerting a toxic effect were excluded from analysis.

Gene silencing. Silencing of Sigmar1 was performed using commercial, specific shRNAs from Sigma-Aldrich. Lentiviral particles expressing shRNAs were produced in 293T cells and used to transduce fibroblasts. Silencing of PERK and UBC were performed using specific siRNAs from SCBT and Dharmacon, respectively.

Immunoblotting and quantification. Protein lysates were run in polyacrylamide gels and probed with the following antibodies: $\alpha$ SMA (DAKO, M0851); tubulin (Sigma-Aldrich, T5168); $\beta$-actin-HRP (Sigma-A1drich, A3854); Sigmar1 (Santa Cruz Biotechnology, sc-137075); p-SMAD2 S465/467 (Cell Signaling Technology, 138D4); PERK (Santa Cruz Biotechnology, sc-377400); Notch1 (Santa Cruz Biotechnology, C-20, sc-6014-R); Hsc70 (Enzo Life Sciences, 1B5); SMAD2/3 (Santa Cruz Biotechnology, sc-133098); anti-Myc epitope (Santa Cruz Biotechnology, 9E10, sc-40); p-PERK (Cell Signaling Technology, p-PERK-Thr980, 16F8); p-eIF2a Ser-51 (Cell Signaling Technology, D9G8, 3398); eIF2A (Santa Cruz Biotechnology, D-3, sc133132); and Smad2 (Cell Signaling Technology, D43B4, 5339). Densitometric analysis of bands was performed using ImageJ software (NIH).

Quantitative real-time PCR. RNA was extracted and purified using TRIzol, followed by cDNA synthesis. Sequences of the primers are provided in Supplemental Table 5.

Calcium imaging. Cells were loaded with the calcium indicator Fluo4 and imaged using a scanning confocal microscope or a microplate reader. In both cases, data were analyzed using the F/F0 method, in which $\mathrm{F}$ represents the fluorescence at any time point and F0 is the mean baseline fluorescence, averaged between either the first 10 frames for confocal imaging or the first 5 frames for multi-plate imaging.

Proliferation assays. For the MTT assay, cells were incubated with the MTT reagent and solubilized for optical signal reading at $570 \mathrm{~nm}$. Alternatively, cells were stained with crystal violet and optical absorbance measured at $590 \mathrm{~nm}$.

Scratch-wound assays. A scratch was made in a cell monolayer and the wound closure was observed for 24 hours, followed by cell staining with crystal violet.

Luciferase reporter assays. Cells were transfected with both CAGA-Luc and GFP plasmids prior to the administration of TGF- $\beta$, haloperidol, and their combination. Luciferase activity was determined using a plate reader.

Collagen gel contraction assay. Fibroblasts were embedded in a collagen gel and transferred to a $37^{\circ} \mathrm{C}$ incubator for 1 hour for gel polymerization. Gels were imaged over the next days.

Animal experiments. MI was performed as previously described (93). Mice were treated with haloperidol at a concentration of $2 \mathrm{mg} / \mathrm{kg}$ i.p. twice per week. Animals were sacrificed at either 10 days or 8 weeks.

A bolus of bleomycin (0.03 U/mouse) was injected into the trachea of COLL-EGFP mice. Haloperidol was administered i.p. twice per week. For the comparative analysis of haloperidol, nintedanib, and pirfenidone in bleomycin-induced fibrosis, drugs were given orally once per day by gavage.

Lung adenocarcinoma LG cells were injected into the tail vein of syngeneic C57BL/6 mice. Haloperidol (2 mg/kg) was administered i.p. twice per week. 
Histology. Immunocytochemistry and immunohistochemistry were performed on fixed cells and tissues. Paraffin-embedded sections of the heart after MI were processed for Masson-Goldner trichrome staining to determine the fibrotic area. The following primary antibodies were used for immunofluorescence: aSMA-Cy3 (Sigma-Aldrich, clone 1A4); vimentin (Cell Signaling Technology, D21H3); Ki-67 (Cell Signaling Technology, D3B5); p-PERK (Cell Signaling Technology, p-PERK-Thr980, 16F8); and calreticulin (BD Biosciences, 612136).

Microscopy and image analysis. Images were acquired with a Leica microscope equipped with a DFC300 camera, a Nikon Eclipse Ti-E inverted fluorescence microscope, and Zeiss LSM 880 and LSM 510 META confocal microscopes. Images were analyzed using ImageJ NIH/Fiji software.

Statistics. Data are expressed as mean \pm standard deviation (SD) or standard error of mean (SEM), as appropriate, and the number of samples per group is indicated in the legends. Statistical analysis for 2 independent data points was determined using 2 tailed $t$ test, and larger data sets were compared using 1-way ANOVA with Kruskal-Wallis post hoc test and multiple comparisons with Dunn's multiple-comparisons test. For analysis of functional data over multiple time points we used 2-way ANOVA for repeated measurements, followed by Bonferroni's or Dunn's post hoc test. All statistical analysis was performed using GraphPad Prism 6.0 or Microsoft Excel 2010/2016. $P$ less than 0.05 was considered significant $\left({ }^{*} P<0.05,{ }^{* *} P<0.01,{ }^{* *} P<0.001\right)$.

Study approval. Animal care and treatment were conducted in conformity with institutional guidelines in compliance with national and international laws and policies. Ethical and experimental procedures were reviewed and approved by the ICGEB Animal Welfare board, meeting the requirements of the EU Directive 2010/63/EU, and by the Italian Ministry of Health (approval number 806/2018-PR).

\section{Author contributions}

M Rehman performed and analyzed most of the cell culture experiments with the help of TB and NR. SV performed and analyzed in vivo experiments, with the contribution of CL, MC, and M Raspa. LB performed and analyzed the high-throughput screening with the help of VM, KV, and TK. FC and SC performed and analyzed the calcium imaging experiments. GR and PC performed and analyzed molecular modeling. CG, MPM, and NS performed mass spectrometry. CC and AM contributed to the experiments on Notch1 and ER stress, respectively. MG and GDS contributed to the setup of all facilities used in the experiments and provided critical evaluations of the results. M Rehman and SZ designed the study, analyzed the results, and wrote the manuscript.

\section{Acknowledgments}

This work was supported by postdoctoral fellowships from ICGEB and Area Science Park (to M Rehman), grant AIRC IG 201619032 (to SZ), grant 14CVD04 from the Leducq Foundation Transatlantic Network of Excellence and grant RF-2011-02348164 "Cardiorigen" from the Italian Ministry of Health (to MG). We are thankful to Maristella Coglievina, Willy De Mattia, Stefano Artico, and Barbara Boziglav for excellent technical assistance.

Address correspondence to: Serena Zacchigna, Cardiovascular Biology Laboratory, ICGEB and University of Trieste, Padriciano, 99, 34149 Trieste, Italy. Phone: 39.040.3757.354/214/357. Email: zacchign@icgeb.org.

1. Murtha LA, et al. The processes and mechanisms of cardiac and pulmonary fibrosis. Front Physiol. $2017 ; 8: 777$.

2. Togo S, Polanska UM, Horimoto Y, Orimo A. Carcinoma-associated fibroblasts are a promising therapeutic target. Cancers (Basel). 2013;5(1):149-169.

3. Kuehl T, Lagares D. BH3 mimetics as anti-fibrotic therapy: Unleashing the mitochondrial pathway of apoptosis in myofibroblasts. Matrix Biol. 2018;68-69:94-105.

4. Parker MW, et al. Fibrotic extracellular matrix activates a profibrotic positive feedback loop. J Clin Invest. 2014;124(4):1622-1635

5. Mack M. Inflammation and fibrosis. Matrix Biol. 2018;68-69:106-121.

6. Khalil H, et al. Fibroblast-specific TGF- $\beta$-Smad2/3 signaling underlies cardiac fibrosis. J Clin Invest. 2017;127(10):3770-3783.

7. Rosenbloom J, Mendoza FA, Jimenez SA. Strategies for anti-fibrotic therapies. Biochim Biophys Acta. 2013;1832(7):1088-1103.

8. Selman M. From anti-inflammatory drugs through antifibrotic agents to lung transplantation: a long road of research, clinical attempts, and failures in the treatment of idiopathic pulmonary fibrosis. Chest. 2002;122(3):759-761.

9. Li X, Zhu L, Wang B, Yuan M, Zhu R. Drugs and Targets in Fibrosis. Front Pharmacol. 2017;8:855.

10. Czaja AJ, Carpenter HA. Decreased fibrosis during corticosteroid therapy of autoimmune hepatitis. J Hepatol. 2004;40(4):646-652.

11. Swartz RD. Idiopathic retroperitoneal fibrosis: a review of the pathogenesis and approaches to treatment. Am J Kidney Dis. 2009;54(3):546-553. 
12. Minicucci MF, Azevedo PS, Polegato BF, Paiva SA, Zornoff LA. Heart failure after myocardial infarction: clinical implications and treatment. Clin Cardiol. 2011;34(7):410-414.

13. de Salvi Guimarães F, et al. Dexamethasone-induced cardiac deterioration is associated with both calcium handling abnormalities and calcineurin signaling pathway activation. Mol Cell Biochem. 2017;424(1-2):87-98.

14. Rafii R, Juarez MM, Albertson TE, Chan AL. A review of current and novel therapies for idiopathic pulmonary fibrosis. $J$ Thorac Dis. 2013;5(1):48-73.

15. Huang S, Frangogiannis NG. Anti-inflammatory therapies in myocardial infarction: failures, hopes and challenges. Br J Pharmacol. 2018;175(9):1377-1400.

16. Fala L. Ofev (nintedanib): First tyrosine kinase inhibitor approved for the treatment of patients with idiopathic pulmonary fibrosis. Am Health Drug Benefits. 2015;8(Spec Feature):101-104.

17. Myllärniemi M, Kaarteenaho R. Pharmacological treatment of idiopathic pulmonary fibrosis - preclinical and clinical studies of pirfenidone, nintedanib, and N-acetylcysteine. Eur Clin Respir J. 2015;2:10.3402/ecrj.v2.26385.

18. Wollin L, Maillet I, Quesniaux V, Holweg A, Ryffel B. Antifibrotic and anti-inflammatory activity of the tyrosine kinase inhibitor nintedanib in experimental models of lung fibrosis. J Pharmacol Exp Ther. 2014;349(2):209-220.

19. Pan J, et al. Inhibition of Bcl-2/xl with ABT-263 selectively kills senescent type ii pneumocytes and reverses persistent pulmonary fibrosis induced by ionizing radiation in mice. Int J Radiat Oncol Biol Phys. 2017;99(2):353-361.

20. Lagares D, et al. Targeted apoptosis of myofibroblasts with the BH3 mimetic ABT-263 reverses established fibrosis. Sci Transl Med. 2017;9(420):eaal3765.

21. Moncsek A, et al. Targeting senescent cholangiocytes and activated fibroblasts with B-cell lymphoma-extra large inhibitors ameliorates fibrosis in multidrug resistance 2 gene knockout (Mdr2\%) mice. Hepatology. 2018;67(1):247-259.

22. Yazdani S, Bansal R, Prakash J. Drug targeting to myofibroblasts: Implications for fibrosis and cancer. Adv Drug Deliv Rev. 2017;121:101-116.

23. Davis J, Burr AR, Davis GF, Birnbaumer L, Molkentin JD. A TRPC6-dependent pathway for myofibroblast transdifferentiation and wound healing in vivo. Dev Cell. 2012;23(4):705-715.

24. Davis J, et al. MBNL1-mediated regulation of differentiation RNAs promotes myofibroblast transformation and the fibrotic response. Nat Commun. 2015;6:10084.

25. Oh RS, et al. RNAi screening identifies a mechanosensitive ROCK-JAK2-STAT3 network central to myofibroblast activation. J Cell Sci. 2018;131(10):jcs209932.

26. Desmoulière A, Geinoz A, Gabbiani F, Gabbiani G. Transforming growth factor-beta 1 induces alpha-smooth muscle actin expression in granulation tissue myofibroblasts and in quiescent and growing cultured fibroblasts. J Cell Biol. 1993;122(1):103-111.

27. Masur SK, Dewal HS, Dinh TT, Erenburg I, Petridou S. Myofibroblasts differentiate from fibroblasts when plated at low density. Proc Natl Acad Sci USA. 1996;93(9):4219-4223.

28. Magness ST, Bataller R, Yang L, Brenner DA. A dual reporter gene transgenic mouse demonstrates heterogeneity in hepatic fibrogenic cell populations. Hepatology. 2004;40(5):1151-1159.

29. Vaglio A, et al. Prednisone versus tamoxifen in patients with idiopathic retroperitoneal fibrosis: an open-label randomised controlled trial. Lancet. 2011;378(9788):338-346

30. van Bommel EF, Siemes C, Hak LE, van der Veer SJ, Hendriksz TR. Long-term renal and patient outcome in idiopathic retroperitoneal fibrosis treated with prednisone. Am J Kidney Dis. 2007;49(5):615-625.

31. Walter N, Collard HR, King TE. Current perspectives on the treatment of idiopathic pulmonary fibrosis. Proc Am Thorac Soc. 2006;3(4):330-338.

32. Mahadik SP, Mukherjee S, Laev H, Reddy R, Schnur DB. Abnormal growth of skin fibroblasts from schizophrenic patients. Psychiatry Res. 1991;37(3):309-320.

33. Morikawa M, Derynck R, Miyazono K. TGF- $\beta$ and the TGF- $\beta$ family: Context-dependent roles in cell and tissue physiology. Cold Spring Harb Perspect Biol. 2016;8(5):a021873.

34. Seeman P. Brain dopamine receptors. Pharmacol Rev. 1980;32(3):229-313.

35. Awouters FH, Lewi PJ. Forty years of antipsychotic Drug research--from haloperidol to paliperidone--with Dr. Paul Janssen. Arzneimittelforschung. 2007;57(10):625-632.

36. Hayashi T, Su T. The sigma receptor: evolution of the concept in neuropsychopharmacology. Curr Neuropharmacol. 2005;3(4):267-280.

37. Schmidt HR, Zheng S, Gurpinar E, Koehl A, Manglik A, Kruse AC. Crystal structure of the human $\sigma 1$ receptor. Nature. 2016;532(7600):527-530.

38. Friesner RA, et al. Glide: a new approach for rapid, accurate docking and scoring. 1. Method and assessment of docking accuracy. J Med Chem. 2004;47(7):1739-1749.

39. Halgren TA, et al. Glide: a new approach for rapid, accurate docking and scoring. 2. Enrichment factors in database screening. J Med Chem. 2004;47(7):1750-1759.

40. Yano $\mathrm{H}$, et al. Pharmacological profiling of sigma 1 receptor ligands by novel receptor homomer assays. Neuropharmacology. 2018;133:264-275.

41. Gueguinou M, et al. The SigmaR1 chaperone drives breast and colorectal cancer cell migration by tuning SK3-dependent Ca ${ }^{2+}$ homeostasis. Oncogene. 2017;36(25):3640-3647.

42. Kubickova J, et al. Haloperidol affects plasticity of differentiated NG-108 cells through $\sigma 1 \mathrm{R} / \mathrm{IP} 3 \mathrm{R} 1 \mathrm{complex}$. Cell Mol Neurobiol. 2018;38(1):181-194.

43. Lytton J, Westlin M, Hanley MR. Thapsigargin inhibits the sarcoplasmic or endoplasmic reticulum Ca-ATPase family of calcium pumps. J Biol Chem. 1991;266(26):17067-17071.

44. Mekahli D, Bultynck G, Parys JB, De Smedt H, Missiaen L. Endoplasmic-reticulum calcium depletion and disease. Cold Spring Harb Perspect Biol. 2011;3(6):a004317.

45. Waser M, Mesaeli N, Spencer C, Michalak M. Regulation of calreticulin gene expression by calcium. J Cell Biol. 1997;138(3):547-557.

46. Lauressergues E, et al. Does endoplasmic reticulum stress participate in APD-induced hepatic metabolic dysregulation? Neuro- 
pharmacology. 2012;62(2):784-796.

47. Schröder M, Kaufman RJ. ER stress and the unfolded protein response. Mutat Res. 2005;569(1-2):29-63.

48. Roti G, et al. Complementary genomic screens identify SERCA as a therapeutic target in NOTCH1 mutated cancer. Cancer Cell. 2013;23(3):390-405

49. Zhang $\mathrm{H}$, et al. NOTCH inhibits osteoblast formation in inflammatory arthritis via noncanonical NF-kB. J Clin Invest. 2014;124(7):3200-3214.

50. Roti G, et al. Leukemia-specific delivery of mutant NOTCH1 targeted therapy. J Exp Med. 2018;215(1):197-216.

51. Ilagan MX, Kopan R. Selective blockade of transport via SERCA inhibition: the answer for oncogenic forms of Notch? Cancer Cell. 2013;23(3):267-269.

52. Noseda M, et al. Smooth muscle alpha-actin is a direct target of Notch/CSL. Circ Res. 2006;98(12):1468-1470.

53. Tang Y, Urs S, Liaw L. Hairy-related transcription factors inhibit Notch-induced smooth muscle alpha-actin expression by interfering with Notch intracellular domain/CBF-1 complex interaction with the CBF-1-binding site. Circ Res. 2008;102(6):661-668.

54. Wang Y, et al. Blocking Notch in endothelial cells prevents arteriovenous fistula failure despite CKD. J Am Soc Nephrol. 2014;25(4):773-783.

55. Shinoda Y, Tagashira H, Bhuiyan MS, Hasegawa H, Kanai H, Fukunaga K. Haloperidol aggravates transverse aortic constriction-induced heart failure via mitochondrial dysfunction. J Pharmacol Sci. 2016;131(3):172-183.

56. Castaing N, Titier K, Canal-Raffin M, Moore N, Molimard M. Postmortem redistribution of two antipsychotic drugs, haloperidol and thioridazine, in the rat. J Anal Toxicol. 2006;30(7):419-425.

57. Sampedro MC, et al. Screening and quantification of antipsychotic drugs in human brain tissue by liquid chromatography-tandem mass spectrometry: application to postmortem diagnostics of forensic interest. Forensic Sci Int. 2012;219(1-3):172-178.

58. Kim CH, Cheng SL, Kim GS. Effects of dexamethasone on proliferation, activity, and cytokine secretion of normal human bone marrow stromal cells: possible mechanisms of glucocorticoid-induced bone loss. J Endocrinol. 1999;162(3):371-379.

59. Kato T, Monji A, Hashioka S, Kanba S. Risperidone significantly inhibits interferon-gamma-induced microglial activation in vitro. Schizophr Res. 2007;92(1-3):108-115.

60. Dygai AM, et al. Antifibrotic and anti-inflammatory activity of a neuroleptic drug on the model of pulmonary fibrosis. Bull Exp Biol Med. 2012;152(6):679-683.

61. Ifteni P, et al. Haloperidol and sudden cardiac death in dementia: autopsy findings in psychiatric inpatients. Int J Geriatr Psychiatry. 2015;30(12):1224-1229.

62. Kriwisky M, Perry GY, Tarchitsky D, Gutman Y, Kishon Y. Haloperidol-induced torsades de pointes. Chest. 1990;98(2):482-484.

63. Hassaballa HA, Balk RA. Torsade de pointes associated with the administration of intravenous haloperidol:a review of the literature and practical guidelines for use. Expert Opin Drug Saf. 2003;2(6):543-547.

64. Hardy JR, O'Shea A, White C, Gilshenan K, Welch L, Douglas C. The efficacy of haloperidol in the management of nausea and vomiting in patients with cancer. J Pain Symptom Manage. 2010;40(1):111-116

65. Hui D, et al. Effect of lorazepam with haloperidol vs haloperidol alone on agitated delirium in patients with advanced cancer receiving palliative care: A randomized clinical trial. JAMA. 2017;318(11):1047-1056.

66. Spruce BA, et al. Small molecule antagonists of the sigma-1 receptor cause selective release of the death program in tumor and self-reliant cells and inhibit tumor growth in vitro and in vivo. Cancer Res. 2004;64(14):4875-4886.

67. Lee JK, Nam DH, Lee J. Repurposing antipsychotics as glioblastoma therapeutics: Potentials and challenges. Oncol Lett. 2016;11(2):1281-1286.

68. Rousseaux CG, Greene SF. Sigma receptors [ $\sigma \mathrm{Rs}]$ : biology in normal and diseased states. J Recept Signal Transduct Res. 2015;36(4):327-388

69. Brune S, et al. The sigma enigma: in vitro/in silico site-directed mutagenesis studies unveil $\sigma 1$ receptor ligand binding. Biochemistry. 2014;53(18):2993-3003.

70. Seth P, et al. Expression pattern of the type 1 sigma receptor in the brain and identity of critical anionic amino acid residues in the ligand-binding domain of the receptor. Biochim Biophys Acta. 2001;1540(1):59-67.

71. Bai T, Wang S, Zhao Y, Zhu R, Wang W, Sun Y. Haloperidol, a sigma receptor 1 antagonist, promotes ferroptosis in hepatocellular carcinoma cells. Biochem Biophys Res Commun. 2017;491(4):919-925.

72. Hayashi T, Su TP. Sigma-1 receptor chaperones at the ER-mitochondrion interface regulate $\mathrm{Ca}(2+)$ signaling and cell survival Cell. 2007;131(3):596-610

73. Tamagnone L, Zacchigna S, Rehman M. Taming the Notch transcriptional regulator for cancer therapy. Molecules. 2018;23(2):431.

74. Röhrl C, et al. Endoplasmic reticulum stress impairs cholesterol efflux and synthesis in hepatic cells. J Lipid Res. 2014;55(1):94-103

75. Contessa JN, Bhojani MS, Freeze HH, Rehemtulla A, Lawrence TS. Inhibition of N-linked glycosylation disrupts receptor tyrosine kinase signaling in tumor cells. Cancer Res. 2008;68(10):3803-3809.

76. Aster JC, Simms WB, Zavala-Ruiz Z, Patriub V, North CL, Blacklow SC. The folding and structural integrity of the first LIN-12 module of human Notch1 are calcium-dependent. Biochemistry. 1999;38(15):4736-4742.

77. Gordon WR, Vardar-Ulu D, Histen G, Sanchez-Irizarry C, Aster JC, Blacklow SC. Structural basis for autoinhibition of Notch. Nat Struct Mol Biol. 2007;14(4):295-300.

78. Rand MD, Lindblom A, Carlson J, Villoutreix BO, Stenflo J. Calcium binding to tandem repeats of EGF-like modules. Expression and characterization of the EGF-like modules of human Notch-1 implicated in receptor-ligand interactions. Protein Sci. 1997;6(10):2059-2071.

79. Tien AC, et al. Ero1L, a thiol oxidase, is required for Notch signaling through cysteine bridge formation of the Lin12-Notch repeats in Drosophila melanogaster. J Cell Biol. 2008;182(6):1113-1125.

80. Hu B, Phan SH. Notch in fibrosis and as a target of anti-fibrotic therapy. Pharmacol Res. 2016;108:57-64.

81. Nistri S, Sassoli C, Bani D. Notch signaling in ischemic damage and fibrosis: Evidence and clues from the heart. Front Pharmacol. 2017;8:187.

82. Liu Y, Huang G, Mo B, Wang C. Artesunate ameliorates lung fibrosis via inhibiting the Notch signaling pathway. Exp Ther Med. 2017;14(1):561-566. 
83. Dees C, et al. Inhibition of Notch signaling prevents experimental fibrosis and induces regression of established fibrosis. Arthritis Rheum. 2011;63(5):1396-1404

84. Nemir M, et al. The Notch pathway controls fibrotic and regenerative repair in the adult heart. Eur Heart J. 2014;35(32):2174-2185

85. Chen Y, et al. Inhibition of Notch signaling by a $\gamma$-secretase inhibitor attenuates hepatic fibrosis in rats. PLoS One. 2012;7(10):e46512.

86. Tanjore H, Blackwell TS, Lawson WE. Emerging evidence for endoplasmic reticulum stress in the pathogenesis of idiopathic pulmonary fibrosis. Am J Physiol Lung Cell Mol Physiol. 2012;302(8):L721-L729.

87. Kropski JA, Lawson WE, Young LR, Blackwell TS. Genetic studies provide clues on the pathogenesis of idiopathic pulmonary fibrosis. Dis Model Mech. 2013;6(1):9-17.

88. Korfei M, et al. Epithelial endoplasmic reticulum stress and apoptosis in sporadic idiopathic pulmonary fibrosis. Am J Respir Crit Care Med. 2008;178(8):838-846.

89. Lawson WE, et al. Endoplasmic reticulum stress in alveolar epithelial cells is prominent in IPF: association with altered surfactant protein processing and herpesvirus infection. Am J Physiol Lung Cell Mol Physiol. 2008;294(6):L1119-L1126.

90. Chow CY, Wang X, Riccardi D, Wolfner MF, Clark AG. The genetic architecture of the genome-wide transcriptional response to ER stress in the mouse. PLoS Genet. 2015;11(2):e1004924.

91. Marciniak SJ, et al. CHOP induces death by promoting protein synthesis and oxidation in the stressed endoplasmic reticulum Genes Dev. 2004;18(24):3066-3077.

92. Dimitrova N, et al. Stromal expression of miR-143/145 promotes neoangiogenesis in lung cancer development. Cancer Discov. 2016;6(2):188-201.

93. Eulalio A, et al. Functional screening identifies miRNAs inducing cardiac regeneration. Nature. 2012;492(7429):376-381. 